\title{
1 Selection for non-specific adhesion is a driver of FimH evolution 2 increasing Escherichia coli biofilm capacity
}

3

4 5

6

Keywords: Biofilm, adhesion, Escherichia coli, experimental evolution

Mari YOSHIDA ${ }^{1}$, Stanislas THIRIET-RUPERT ${ }^{1}$; Leonie MAYER ${ }^{1}$, Christophe BELOIN*1 and Jean-Marc GHIGO*1

${ }^{1}$ Unité de Génétique des Biofilms, Institut Pasteur, UMR CNRS2001, Paris, France

*Co-Corresponding authors: jean-marc.ghigo@pasteur.fr, christophe.beloin@pasteur.fr

Running title: Evolution of Escherichia coli FimH adhesin increases biofilm capacities. 


\section{ABSTRACT}

Bacterial interactions with surfaces rely on the coordinated expression and interplay of surface exposed adhesion factors. However, how bacteria dynamically modulate their vast repertoire of adhesins to achieve surface colonization is not yet well-understood. We used experimental evolution and positive selection for improved adhesion to investigate how an initially poorly adherent Escherichia coli strain increased its adhesion capacities to abiotic surfaces. We showed that all identified evolved clones acquired mutations located almost exclusively in the lectin domain of $\operatorname{fim} H$, the gene coding for the $\alpha$-D-mannose-specific tip adhesin of type 1 fimbriae. While most of these fim $H$ mutants showed reduced mannosebinding ability, they all displayed enhanced binding to abiotic surfaces, indicating a trade-off between FimH-mediated specific and non-specific adhesion properties. Several of the identified mutations were already reported in FimH lectin domain of pathogenic and environmental E. coli, suggesting that, beyond patho-adaptation, FimH microevolution favoring non-specific surface adhesion could constitute a selective advantage for natural $E$. coli isolates. Consistently, although E. coli deleted for the fim operon still evolves an increased adhesion capacity, mutants selected in the $\Delta$ fim background are outcompeted by 5 fim $H$ mutants revealing clonal interference for adhesion. Our study therefore provides insights 6 into the plasticity of $E$. coli adhesion potential and shows that evolution of type 1 fimbriae is a 7 major driver of the adaptation of natural E. coli to colonization. 


\section{INTRODUCTION}

Adhesion and subsequent formation of biofilms on environmental or host surfaces enable bacteria to withstand natural mechanical fluxes and is an essential step of most colonization or infection processes ${ }^{1-5}$. In commensal and pathogenic Escherichia coli, adhesion and biofilm formation is achieved using a large arsenal of adhesion factors ${ }^{2,4}$, including proteinaceous adhesins protruding from the bacterial cell envelope and promoting non-specific surface adhesion (e.g. amyloid curli), afimbrial autotransporter adhesins mediating aggregation via homotypic self-interactions (e.g. Ag43 adhesin) or polymeric fimbrial adhesins that specifically recognize oligosaccharidic ligands (e.g. type 1 fimbriae) ${ }^{6-10}$. E. coli also secretes several polysaccharidic exopolymers such as cellulose, $\beta-1,6-N$-acetyl-D-glucosamine polymer and capsular polysaccharides, promoting or hindering adhesion on living or inert surfaces ${ }^{11-15}$. In addition to these well characterized surface structures, the E. coli genome contains several partially characterized genes or operons encoding potential adhesion factors 6,7,16,17. The expression of $E$. coli adhesins is controled by diverse regulatory networks enabling rapid transcriptional change in response to stresses and environmental cues such as oxygen levels, $\mathrm{pH}$ and chemical gradients ${ }^{6,18-20}$. However, how E. coli controls its adhesive properties and coordinates the interplay between its adhesion factors, while avoiding antagonistic physical interferences between adhesins is still poorly understood. Here, we used a dynamic in vitro biofilm model amenable to experimental evolution to explore how E. coli adapts when subjected to positive selection for increased capacity to bind to an abiotic surface and form biofilms. We showed that, despite the diversity of E. coli adhesins, all end-point populations displaying increased biofilm biomass after positive selection almost exclusively acquired mutations in $\operatorname{fim} H$, the gene coding for the $\alpha$-Dmannose-specific tip adhesin of type 1 fimbriae enabling adhesion to mannosylated epithelial cells. The identified fim $H$ mutations, including mutations found in environmental and clinical $E$. coli isolates, displayed enhanced capacity for initial adhesion to abiotic surfaces but reduced mannose-binding properties and outcompeted the strongest biofilm-forming mutant emerging from a selection performed in a strain lacking the whole fim operon. This indicates that selection of $f i m H$ mutants with increased non-specific adhesion capacity could provide commensal or pathogenic E. coli a selective advantage for surface colonization and persistence in the environment. Our study therefore provides direct insights into the plasticity of the E. coli adhesion repertoire and shows that biofilm formation is a powerful driver of the evolution of E. coli adhesion potential. 


\section{RESULTS}

\section{Positive selection of Escherichia coli mutants with increased biofilm capacity}

We subjected the poor biofilm-forming strain E. coli MG1655 to positive selection for spontaneous mutants with increased adhesion capacity using continuous-flow biofilm microfermenters (Fig. 1A). We conducted 12 parallel evolution experiments with 15 cycles of 8 hours biofilm formation followed by overnight planktonic growth (15 days). The capacity of biofilm formation in microfermenters was enhanced for 6 of the 12 evolved population, with a 10 to 100 -fold increase for 4 of them as compared to the WT ancestor (G6, R4, R5, R6) (Fig. 1BC and Supp. Fig. S1A). These 6 populations also displayed an enhanced capacity to form biofilms in microtiter plate (Supp. Fig S1B) showing that the evolved trait was robust over two different biofilm models. To study the dynamic of the evolution of the adhesion capacities of the different population we performed microtiter plate biofilm assays for each cycle of the different population (Fig. 1D). Biofilm formation started to increase at cycle 5 for the G6 population and around cycle 10 for the population G1, G3, R4, R5 and R6 (Fig. 1D). Consistently, the enhanced biofilm formation evolved trait was also observed at clones level since heterogeneous but significant increase in biofilm capacity compared to the ancestral WT strain was shown in 15 individual clones isolated from each of the 6 more adherent populations (Fig. 1E and Supp. Fig.S1B).

\section{End-point biofilm-positive mutants carry mutations in the gene encoding the type 1}

\section{fimbriae tip adhesin FimH}

To identify the nature of the mutations leading to increased adhesion, we sequenced and compared the ancestral population to the genome of eight populations at the end of 15 selection cycles, including the six biofilm populations with enhanced biofilm forming capacity (G1, G3, G6, R4, R5, R6) and six populations that showed moderate or no biofilm increase (G2, G4, G5, R1, R2 and R3) (Supp. Fig. S1B). We also sequenced four individual clones isolated from each of the six biofilm positive populations and two of the six biofilm negative populations. The populations and clones with enhanced biofilm capacities all carried mutations at high frequencies located in the gene coding for FimH and no fim $\mathrm{H}$ mutation was detected in the six populations showing no increase in biofilm capacity after 15 cycles of positive selection (Fig. 2A and Supp. Table S1). FimH is the D-mannose specific adhesin of type 1 fimbriae, a major $E$. coli adhesin enabling epithelial cell colonization and shown to be critical for biofilm formation on abiotic surfaces ${ }^{21}$. FimH is a 300 residue and $31.473 \mathrm{kDa}$ 
protein consisting of a pilin domain (amino acid 181-294) and a mannose-binding lectin domain (amino acid 23-177) connected through a 4 amino acid linker peptide chain (Fig. 2B).

We identified 3 in-frame deletions ( $\Delta 29-40$ and $\Delta 64-70$, and $\Delta 89-139)$ and three nonsynonymous substitutions (V77A, L79P, G82C) in the lectin domain of the FimH protein, as well as one non-synonymous substitution (S16L) in the signal peptide of the FimH protein

114 (Fig. 2BC). Some fimH mutations coexisted within populations: L79P and G82C were found in R5 population, V77A and $\Delta 89-139$ in R6 population, and $\Delta 29-40$ and $\Delta 64-70$ in G6 population. By contrast, R4 population only had the S16L substitution, and G1 and G3 populations only had V77A mutations (Supp.Table S1).

118 In order to check if this outcome was specific to the parameters used in our selection protocol,

119 we ran another experiment using different selection pressures with less selection cycles (4

120 instead of 15) and longer residence time in biofilm microfermenters at each cycle (18 hours

121 instead of 8 hours). This protocol also resulted in the selection of $f i m H$ mutation (G87C),

122 emphasizing the predominant role of this adhesin in enhanced binding capacities and biofilm

123 formation (Fig. 2BC). In order to determine the dynamics of emergence and population

124 spread of the identified fim $H$ mutations over time, we performed a Sanger sequencing analysis

125 of fimH region PCR products of populations collected at each of the 15 positive selection

126 cycles that led to increased biofilm capacities (Fig. 2D). All fim H in-frame deletions and the

127 G82C mutation emerged as early as the fourth/fifth cycle, while the other non-synonymous

128 FimH substitutions emerged at cycle 6 or later.

\section{The phenotypic differences between fim $\mathrm{H}$ mutants are not due to phase variable}

\section{expression of the fim operon}

132 We isolated individual clones carrying an independent mutation in FimH and measured their

133 biofilm capacity using continuous biofilm microfermenters. While most tested evolved clones

134 showed close to wild-type growth rate, three mutations (V77A, L79P and $429-40$ ) slightly

135 impacted growth (Supp. Fig. S2AB) and 1:1 competition between these 3 clones and WT

136 confirmed this fitness default (Supp. Fig. S2C). All clones but the one carrying L79P,

137 demonstrated increased biofilm-forming capacity, i.e. 1.8 to 113 times more than WT

138 ancestral strain after 24 hours of incubation in microfermenters (Fig. 3A). The mutants

139 encoding FimH deletions $\Delta 64-70$ and $\Delta 89-139$ displayed the highest biofilm formation,

140 relative to the ancestral WT (Fig. 3B). This enhanced capacity to form biofilms could be

141 linked to phase variable expression of the fim operon that enable rapid on/off change of type 1 
142 fimbriae production by inversion of the fimA promoter mediated by FimB and FimE

143 recombinases (see fim region in Supp. Fig. S3A) ${ }^{22-24}$. However, a test of the orientation of the

144 fim promoter by PCR did not reveal any significant differences in ON/OFF status between

145 wild type parental and the evolved clones. The mutant clones were mainly OFF in planktonic

146 culture and mainly $\mathrm{ON}$ in biofilm on microfermenter spatula, while none of the clones or the

147 evolved populations displayed any mutations known to impact the fim switch, thus reflecting

148 an enrichment for fim ON status in biofilm conditions (Supp. Fig. S3BC).

\section{Evolved fimH mutations increase non-specific initial adhesion}

151 To characterize the phenotypic consequences of the identified fim $H$ mutants in planktonic

152 conditions and, considering the ON fim status of clones selected in biofilm conditions, we

153 placed the WT and mutant fim operon (and therefore corresponding native or mutated fimH

154 genes) under the control of the lambda PcL constitutive promoter ${ }^{6}$. This led to normalized

155 type 1 fimbriae expression, as shown using immunodetection with anti-FimA antibodies

156 (Supp. Fig. S4). Biofilm biomass formed in microfermenters of PcL-fimH mutants, compared

157 to PcL-fimH WT, showed differences similar to those observed between (non-PcL) mutants

158 and WT strains (compare Fig. 4A with Fig. 3), suggesting that, independently of fim phase

159 variation, the selected FimH mutants displayed specific properties enhancing E. coli biofilm

160 formation. Interestingly, selected mutants out-competed the wild-type strain for increased

161 initial adhesion to glass in 1:1 competition between WT PcL-fimH and mutant PcL-fimH (Fig.

162 4B). This fitness advantage suggests that increased initial non-specific adhesion is one of the

163 main drivers of the enhanced capacity to form biofilms of the fim $\mathrm{H}$ mutant compared to the

164 wild-type ancestral strain. However, we detected a negative correlation between fim $H$ mutants

165 initial adhesion capacity and their biofilm capacity (Spearman's rho $=-0.76$, p-value $=0.037$,

166 Supp. Fig. S5), suggesting a trade-off between initial adhesion and biofilm maturation.

167 We also evaluated the ability of PcL-fimH mutants to bind mannosylated proteins using a

168 yeast agglutination assay performed under static or agitated conditions, where the latter

169 condition promotes catch-bond adhesion ${ }^{25}$. In both situations, all FimH mutations, except

$170 \mathrm{G} 87 \mathrm{C}$, reduced yeast agglutination capacity, with some mutations displaying almost no

171 agglutination, very similar to a $\Delta$ fimH strain agglutination phenotype (Supp Fig. S6). This

172 indicates a clear trade-off between FimH-enhanced adhesion to abiotic surfaces and FimH

173 adhesion to mannose.

175 Mutations identified in FimH are also found in natural and clinical isolates. 
In order to assess whether the FimH mutations identified in our in vitro evolution experiments reflect evolutionary paths occurring in natural or clinical $E$. coli isolates, we retrieved all $E$. coli FimH sequences available in NCBI protein databank as well as 2067 sequenced genomes of E. coli (Supp. Table S2). The resulting NCBI dataset composed of 3266 sequences (Supp. Table S3A) shows a strong bias toward strains of human and animal origin (53.25\% versus $1.81 \%$ for environmental strains, while no information is available for the remaining $45 \%$ ), likely due to the predominant representation of health-related studies. To partly compensate for this skew, we added the fimH sequence of $277 \mathrm{E}$. coli environmental strains from a recent study ${ }^{26}$ (see Methods section and Supp. Table S3B). When the 3543 total FimH protein sequences extracted from this dataset were compared to MG1655 FimH, 10.4 \% (369 FimH sequences) displayed amino-acid changes at the same positions of at least one of the eight mutations identified in our study (Supp. Table S3AB). An enrichment for strains belonging to the B1 phylogroup was identified in these 369 sequences, relative to the whole dataset (Supp. Table S4A). Interestingly, 64 FimH proteins showed mutations that were also identified in our study (one S16L, 18 V77A, one L79P, 42 G87C and two $429-40$ ). The G87C mutation (=G66C when amino acid numbering starts after the signal peptide), a position previously reported to be under positive selection in uropathogenic $E$. coli strains, being the most widespread ${ }^{27,28}$. These results demonstrate that our study recapitulated some of the selection events driving natural evolution of FimH (Fig. 5A). The enrichment in B1 strains previously identified was not maintained in these 64 sequences, this phylogroup being rather depleted relatively to the whole dataset (Supp. Table S4A). However, there was an enrichment for B2 and $\mathrm{F}$ strains (to which most ExPEC strains belong), suggesting that our experimental evolution reflects part of the selective pressures applied to ExPEC strains.

\section{FimH mutational landscape diversity highlights its potential functional plasticity}

All selected mutations in our study were found in the FimH lectin domain and none in its pilin domain. To test whether this was a general trend in FimH sequences or rather specific to our experimental settings, we screened all available FimH sequences for single amino-acid changes and calculated their frequency for each structural part of the FimH sequence (the signal peptide, the lectin domain, the four amino-acids linker and the pilin domain). The majority of these mutations were found in the lectin domain and were significantly higher (1.47-fold, One-way Anova: $\mathrm{df}=1, \mathrm{p}$ value $<2.2 \mathrm{e}-16)$ than expected, while the pilin domain accumulated significantly less mutations (-2.31-fold, One-way Anova: $\mathrm{df}=1, \mathrm{p}$ value $<2.2 \mathrm{e}-$ 16) (Fig. 5B). To analyze the E. coli FimH mutational landscape in more details, the same 
analysis was carried out for each position of the sequence and 20 of them were found to be statistically more prone to mutation (Fig. 5B and Supp. Table S4B). Interestingly, some of the mutations identified at these hotspots were found to co-occur in the same FimH sequences (Supp. Fig. S7). Different phylogroup enrichments were also identified at these polymorphic sites (Supp. Table S4C).

To bring further insight into the evolution of FimH sequences in E. coli, we built a phylogenetic tree (Supp. Fig. S8) and analyzed it with the codeml program in PAML v4.9 ${ }^{29}$.

218 For this purpose, we divided the tree into five sub-groups ( $\mathrm{Sg} 1$ to Sg5) based on phylogroups distribution along the tree (see methods). Overall, the evolutionary analysis showed a prevalence for purifying selection (Supp. Table S5A). However, sequences belonging to Sg3 showed signs of episodic positive selection. Interestingly, most of the sites identified to be positively selected in this group were located in the lectin domain including positions 82 and 87 corresponding to evolved sites in our experimental evolution (Supp. Table S5 AB). Moreover, Sg3 was enriched in strains belonging to phylogroup B2 (Supp. Table S4D) as well as comprised all sequences having amino acids Ser-70 and Asn-78 (Ser-91 and Asn-99 in this study) described to be related to UPEC strains ${ }^{27}$. Finally, a site partition analysis revealed that, although being both under purifying selection, the lectin and the pilin domains underwent a different evolutionary path in Sg3 and Sg5 (Supp. Table S5CD). Overall, these results demonstrate that the lectin domain of FimH is the most subjected to mutations both in laboratory evolved strains and in natural and clinical isolates, which could reflect complementary selection pressure in environments where bacteria need to withstand natural fluxes and perturbations.

In absence of $\mathrm{fimH}$, evolution towards biofilm formation involves a broader range of

\section{mutations}

236 Despite the diversity of E. coli K-12 surface structures known to contribute to biofilm 237 formation ${ }^{30}$, mutations leading to increased biofilm formation revealed by our study were 238 surprisingly targeted to $\mathrm{fim} H$. To test the evolution towards increased adhesion capacity in 239 absence of $f i m H$, we deleted the whole fim operon (fimABCDEFGH see Supp. Fig. S3A) and 240 we ran 12 parallel positive selection experiments of the $\Delta$ fim strain for 15 cycles. We 241 observed an evolution towards significant and relevant increased biofilm formation in both 242 biofilm models for 3 out of $12 \Delta$ fim populations, ${ }^{\Delta} \mathrm{G} 2,{ }^{\Delta} \mathrm{G} 5$ and ${ }^{\Delta} \mathrm{R} 4$ (Fig. 6A-B), with ${ }^{\Delta} \mathrm{G} 5$ 243 and ${ }^{\Delta} \mathrm{R} 4$ achieving 14- and 71-times increased biofilm capacity in biofilm microfermenters 
244 (Fig. 6A). The analysis of the mutations found in these three biofilm-positive evolved $\Delta$ fim

245 populations and in corresponding clones revealed mutations affecting biofilm associated

246 functions such as chaperone-usher fimbrial surface structure (yqiG, ecpR and ecpD),

247 autotransporter adhesin (ycgV, flu/agn43) and flagellum (flgH, ecpR) (Supp. Table S6).

248 Whereas the ecp operon is not expressed in E. coli $\mathrm{K} 12 \mathrm{MG} 1655^{31}$, biofilm-promoting

249 mutations in ecpD were only found in clones also displaying an IS insertion right after ecpR,

250 the regulator of the operon. This IS insertion resulted in ecp operon expression (Supp. Fig.

251 S9), potentiating further mutations in ecpD, leading to increased biofilm formation in $\Delta$ fim

252 population ${ }^{\Delta} \mathrm{R} 4$. These results therefore showed that, in absence of type 1 fimbriae, different

253 mutations in surface structure and adhesins could lead to increased biofilm capacity.

255 fim $H$ mutants outcompete mutants selected in the absence of $f i m H$.

256 To investigate the potential origin of the lack of mutation diversity in evolution experiments

257 performed in the WT background compared to those performed in a $\Delta$ fim background, we

258 reconstructed the ecpD/ecpR mutant from the ${ }^{\Delta} \mathrm{R} 4$ population (the highest biofilm-forming

259 population from the $\Delta$ fim evolution) in a WT background. This mutant was then used in

260 competition assays against the natural fim $H$ mutants with highest biofilm capacity $(\Delta 64-70$

261 and $\Delta 89-139)$ and increased, but lower biofilm capacity (V77A). These competition

262 experiments were performed for three critical steps of our selection protocol: initial adhesion

263 on the glass spatula inserted in biofilm microfermenters, biofilm formation and growth in

264 liquid culture. We showed that, during biofilm formation in microtiter plates as well as

265 growth in liquid culture, both $\Delta 64-70$ and $\Delta 89-139$ fim $H$ mutants outcompeted the ecpD/ecpR

266 mutant (Fig. 6 C). Whereas there was no advantage of the V77A mutation in these conditions,

267 competition for initial adhesion showed a very clear advantage for the V77A fimH mutant

268 and, in a lesser extent, for both $\Delta 64-70$ and $\Delta 89-139$ mutants (Fig. 6 C). These observed

269 fitness advantages of the fim $H$ mutants could account for the out-competition of other adhesin

270 mutations in our evolution experiments. 


\section{DISCUSSION}

In this study, we showed that despite the vast arsenal of proteinaceous and macromolecular surface structures known to contribute to biofilm formation in Escherichia coli, in vitro experimental evolution selecting for increased biofilm-forming capacities systematically led to the acquisition of mutations in the type 1 fimbriae tip adhesin gene $f i m H$, one of the first $E$. coli appendages implicated in biofilm formation on abiotic surfaces ${ }^{21}$. Although the restricted mutational landscape revealed by our study suggests that type 1 fimbriae are the main E. coli adhesins in these experimental conditions, we cannot exclude that mutations in other genes could have emerged and been counterselected before the last selection cycles or been present below the 5\% threshold detection of our breseq analyses. For instance, the G2 population showed an episodic increase in adhesion capacity at day 11 that was not detected afterwards (Fig. 1B). This correlated with the presence of an in-frame deletion in $f l u$, the gene coding for the self-recognition E. coli adhesin Antigen $43^{32,33}$. We hypothesize that this mutation and other potential fim $H$-independent mutants could be outcompeted by emerging fim $H$ mutations leading to a strong bias toward type 1 fimbriae mutations. In support of this hypothesis, we showed that, although positive selection for increased adhesion in a strain deleted for the fim $A-H$ operon also identified mutations in genes encoding adhesins, the strongest biofilmformer amongst these mutants was outcompeted by fim $\mathrm{H}$ mutants for growth, initial adhesion and biofilm formation. In addition, we observed that mutation of the tip-pilus adhesin ecpD was always observed together with the insertion of an IS element that increases expression of the otherwise cryptic ecp pilus operon. This suggests that adhesin expression may first need to be unlocked before productive mutations could enhanced their adhesive properties and our selection could favor bacteria in which fim expression was locked ON, so more likely to evolve towards increased adhesion.

FimH is an allosterically regulated mannose-binding protein and FimH-dependent mannosebinding is considered as important for adhesion to mannosylated cell surface receptors in vivo as well as in vitro biofilm maturation possibly via the recognition of mannose-rich biofilm matrix component ${ }^{34}$. In presence of shear forces, FimH mannose binding-capacity is enhanced by catch-bond mechanisms ${ }^{25,35}$, which could provide a selective advantage in the turbulent conditions used to select for increased-adhesion mutants. However, while most identified fim $H$ mutants displayed an enhanced capacity for initial adhesion to abiotic surface 
encompassing part of FimH mannose-binding pocket were amongst the strongest biofilmformers. This shows that FimH-dependent mannose-binding and catch bond do not significantly contribute to the increased biofilm capacities of the mutants identified in our study. FimH was early demonstrated to contribute to both specific and non-specific adhesion ${ }^{21}$ and our results show that the lectin domain of FimH contributes to both types of adhesion, with residues of this lectin domain directly engaged in interaction with abiotic surfaces. This revealed a trade-off between attachment to mannose and abiotic surfaces that could be due to the fact that residues that are mutated or deleted in our selected fim $\mathrm{H}$ mutants are either at the vicinity of the mannose binding pocket and could directly impact the specificity of the interaction through structural modification (Supplementary Fig. S10). Hydrophobic interactions also play a key role in bacterial adhesion ${ }^{36}$ and mutations reducing hydrophobicity could enhance FimH interactions to abiotic surfaces. Such mutations were selected during our evolution experiments: substitution of hydrophobic by hydrophilic amino acid residue (V77A, L79P), producing the mutants with the highest initial adhesion on the spatula (Fig. 5), substitution of non-polar by polar amino-acid (G82C and G87C) or deletion of stretches of hydrophobic residues ( $\Delta 89-139$ correspond to the deletion of 38 hydrophobic residues out of 51). Hence, increasing FimH hydrophilicity could contribute to improve adhesion of FimH mutants on microfermenter glass spatula. In the case of the S16L mutant in

325 FimH signal peptide, alteration of signal peptide could increase FimH transport efficiency and type 1 fimbriae exposition ${ }^{37}$. However, we could not detect any differences in the quantity of surface exposed FimA in this FimH S16L mutant. Alternatively, the selected mutations in fim $H$ could improve surface contact and adhesion due to changes in the tertiary structure of FimH (Supplementary Fig. S10).

Unexpectedly, our study also revealed a negative correlation between the strength of initial adhesion displayed by the evolved fim $\mathrm{H}$ mutants and their capacity to form mature biofilms. This trade-off is particularly clear in the case of mutations at position V77A and L79P,

334 leading to strong increase in initial adhesion with almost no positive impact on biofilm

335 formation capacity. By contrast, the $\Delta 64-70$ and $\Delta 89-139$ mutations showed low gain in initial adhesion but led to strong increase in biofilm formation. These results illustrate the complexity of the mechanisms at play during biofilm formation. Strong initial adhesion could

338 deeply impact bacterial metabolism and significantly delay biofilm maturation ${ }^{38}$.

339 Alternatively, but these hypotheses are not mutually exclusive, a too strong attachment to the 340 surface might not be optimal to favor later cell-to-cell interaction and matrix production, but 
also the dynamism, including movement of cells, that might be necessary during biofilm maturation.

344 Type 1 fimbriae were shown to contribute to both pathogenic and commensal E. coli colonization of biotic surfaces. FimH-mediated adhesion enables commensal E. coli to adhere to buccal and intestinal epithelia as part of the normal bacterial flora but also enables pathogenic strains to colonize various mannosylated tissues ${ }^{39-41}$. Although mutations in the FimH pilin domain are likely negatively selected because of its critical functional role, the mannose lectin domain displays a higher genetic plasticity, which could lead to functional diversity, conferring selective advantage by modifying and diversifying substrate binding capacity ${ }^{42,43}$. Consistently, several phenotypic variants of the FimH lectin domain have been identified in clinical urinary or intestinal $E$. coli isolates ${ }^{28,44-48}$. These variants are often single point, non-synonymous amino acid substitutions found in the lectin domain but not affecting the mannose-binding pocket directly. While adaptive mutations identified in our study were also found in the lectin domain of the protein, only position 87 (G87C) has been previously described in pathoadaptive variants ${ }^{28,44,45,48}$ and described as a mutational hotspot with amino acid changes to arginine, alanine, serine and cysteine ${ }^{28}$. The G87S and G87C variants are the only mutants that display moderate increases in mannose-binding, but still display catch-bond properties under flow conditions ${ }^{28}$.

While FimH variants previously identified in clinical isolates were mostly associated to tissue tropism ${ }^{28,45,46,48}$, our study shows that some mutations isolated in natural and clinical strains can also be selected for increased initial adhesion to abiotic surface and, as a consequence, biofilm formation capacity. This suggests that evolution of FimH in natural and/or clinical environments can also be driven by abiotic environmental selection pressures. The observation that FimH sequence distribution does not strictly reflect phylogroup clustering (Supp. Fig. S7) further suggests that this gene is subjected to selection pressures specific to each strain environment and, consequently, the need for specific adaptations. Supporting this idea, the mutational landscape analysis of FimH sequences in E. coli revealed 20 hotspots constituting a potential source of functional diversity. These different hotspots have various phylogroup enrichment suggesting that these mutations are related to diverse ecological contexts and environments. For instance, the co-occurrence of S91N and N99S related to UPEC strains ${ }^{27}$ was also identified in environmental strains, which is consistent with the 
49. Although the frequency of this lifestyle switch and how long each E. coli clone resides in the gut and in the environment is not well known, E. coli is able to grow, evolve and adapt in both of these environments. Interestingly, conditions in the mammalian gut are relatively stable as compared to the one found in the outside host environment, and experimental evolution of $E$. coli in the gut did not select for mutation in fim $^{50-54}$, in contrast with studies on plant-associated E. coli demonstrating an evolution towards increased capacity to form biofilm in this environment ${ }^{55}$. Hence, rapidly changing conditions encountered by $E$. coli outside of the host could impose strong selection pressures on environmental E. coli, potentially leading to their rapid evolution ${ }^{56,57}$. The fact that FimH mutations selected in our experiment correspond to naturally occuring mutations enriched in B2 and F phylogroups (mainly ExPEC strains), suggests that mutations favouring non-specific versus specific adhesion could be selected even in strains known for their tissue tropism. One can therefore speculate about the nature of the main in vivo and/or environmental selection pressure driving the evolution sequenced $E$. coli clones. Unfortunately, the clear lack of environmental E. coli strains in the databases hinders genomic analysis. Indeed, among the 3543 FimH sequences included in our analysis 1256 were of unknown origin, 1192 were isolated from human, 690 from animals, 64 from food-related samples and only 341 from the environment. This therefore emphasizes the need for an increased number of sequenced and annotated genomes of environmental E. coli strains.

From an evolutionary perspective, the benefits of FimH mutations affecting mannose binding capacity greatly depend on the environmental surfaces colonized by E. coli. FimH variants with increased mannose affinity under shear stress could be advantageous for bladder colonization, while decreased concentrations of free soluble mannose in the intestine could impose a very different selection pressure. Our findings show that fim $H$ can undergo rapid microevolution, leading to increased non-specific adhesion and biofilm formation independently of mannose-binding capacity. This extended FimH mutational landscape in biofilms could reflect a natural strategy to diversify $E$. coli surface binding capacity, resist physical and chemical disruptions and potentially providing selective advantage for persistence during E. coli cycling between hosts and the environment. 


\section{MATERIAL AND METHODS}

\section{Bacterial strains and growth conditions.}

410 Bacterial strains used in this study are listed in supplementary Table S7. E. coli was grown in

411 M63B1 minimum medium supplemented with glucose $(0.4 \%)$ and kanamycin $(20 \mu \mathrm{g} / \mathrm{mL})$,

412 and incubated at $37^{\circ} \mathrm{C}$. All media and chemicals were purchased from Sigma-Aldrich.

\section{Strain construction.}

415 The WT strains used in the experiments originated from an E. coli MG1655 K12 wild-type

416 strain and were either tagged, at the lambda att site, with red (mars) or green fluorescent

417 protein encoding genes (gfpmut3)(RFP/GFP) by P1 vir transduction. Strains without the

418 whole fim operon ( $\triangle$ fimABCDEFGH) were constructed by $\mathrm{P} 1$ vir phage transduction method

419 from MG1655__fimAICDFGH:: at $^{6}$ into the WT strains. The constitutive promoter

420 controlling the fim operon (PcLfim) ${ }^{7}$ was transferred by lambda-red recombination into WT

421 strains and individual fim mutants using pKOBEGA plasmid and lambda red recombination.

422 The ecpD/ecpR mutant was reconstructed using P1 vir phage transduction into the MG1655

423 K12 wild-type strain tagged with either red (mars) or green fluorescent protein ( $g f p m u t 3$ ).

424 Primers used for genetic contruction are listed in supplementary Table S8.

\section{Biofilm formation in microfermenters.}

427 Continuous-flow biofilm microfermenters containing a removable glass spatula were used as described in ${ }^{58}$, with internal agitation provided by filter-sterilized air-bubbling. Biofilm microfermenters were inoculated by placing the spatula in a culture solution adjusted to $\mathrm{OD}_{600}=1.0$ (containing $5.0 \times 10^{8}$ bacteria $/ \mathrm{mL}$ ) for $10 \mathrm{~min}$. The spatula was then reintroduced into the microfermenter and biofilm culture was performed at $37^{\circ} \mathrm{C}$ in $\mathrm{M} 63 \mathrm{~B} 1$ with $0.4 \%$ glucose. Flow rate was then adjusted $(30 \mathrm{~mL} / \mathrm{h})$ so that total time for renewal of microfermenter medium was shorter than bacterial generation time, thus minimizing planktonic growth by constant dilution of non-biofilm bacteria.

\section{Positive selection procedure.}

437 At the beginning of the positive selection procedure (Day 0) $-80^{\circ} \mathrm{C}$ glycerol stocks of the 438 parental E. coli GFP- or RFP-tagged strains were inoculated in culture tubes containing LB 439 medium supplemented with kanamycin $(20 \mu \mathrm{g} / \mathrm{mL})$ for over-day $8 \mathrm{~h}$ culture, then transferred 440 into M63B1 minimum medium supplemented with $0.4 \%$ glucose and kanamycin for 
overnight 15 h culture. Six removable glass spatula were submerged into the GFP- overnight culture adjusted to $\mathrm{OD}_{600}=1.0$ (ca. $5.0 \times 10^{8}$ bacteria $/ \mathrm{mL}$ ) for $10 \mathrm{~min}$, and six others with the RFP- overnight culture. Each spatula was then reintroduced into one microfermenter and the bacteria adhered to the spatula were incubated for eight hours, during which fresh medium (M63B1 minimum medium supplemented with $0.4 \%$ glucose) flowed $(30 \mathrm{~mL} / \mathrm{h})$ in the microfermenter, constantly diluting non-adhering, planktonic bacteria. After the eight hour incubation in the microfermenters, bacteria that developed as a biofilm onto the spatula were resuspended in culture tubes containing fresh minimum growth medium supplemented with glucose and kanamycin, and cultured overnight. A fraction of each overnight culture was used to prepare a glycerol stock that could be analyzed afterwards and the rest was then used for the inoculation for the next cycle of the positive selection experiment.

Microfermenters were sterilized using 70\% ethanol overnight after each positive selection cycle, then air dried prior to the following positive selection cycle. The sterility of the microfermenters was tested by plating out an aliquot of the fresh medium added to the microfermenters at the beginning of each positive selection cycle. The spatula was autoclavesterilized, machine-washed, then sterilized again between the cycles. During the 15 days of the positive selection procedure, aliquots of the population were stocked at $-80^{\circ} \mathrm{C}$ (glycerol stock) and the adhesion capacity of the populations was monitored by running crystal violet microtiter plate biofilm assay. At the end of the positive selection cycles, bacteria were resuspended from the spatula and these selected end-point populations were stocked. The biofilm formation capacity of these end-point populations were compared to those of nonevolved parental wild type GFP- or RFP-tagged strain after $24 \mathrm{~h}$ in microfermenters by monitoring microfermenters and spatula and biomass after resuspension in M63B1 (no glucose) and determination of $\mathrm{OD}_{600}$ optical density. These $24 \mathrm{~h}$ microfermenter experiments were performed at least three times.

\section{Biofilm microtiter plate assay CV assay}

The glycerol stocks of the target strains or populations were inoculated in LB medium supplemented with kanamycin $(20 \mu \mathrm{g} / \mathrm{mL})$ for over-day $5 \mathrm{~h}$ culture at $37^{\circ} \mathrm{C}$, then inoculated in either a polystyrene Greiner 96-well plate or a polyvinyl chloride Corning plate at $\mathrm{OD}_{600}=$ $0.01\left(5 \times 10^{6}\right.$ cells $\left./ \mathrm{mL}\right)$ in M63B1 minimum medium supplemented with $0.4 \%$ glucose and kanamycin. Incubation was done at $37^{\circ} \mathrm{C}$ for overnight, 16 hours. The supernatant was removed from each well and Bouin solution (Sigma) was applied for 15 minutes to fix the 
biofilm attached to the well. Next the fixation solution was washed 3 times with water and biofilm was stained with 1\% crystal violet solution (QCA) for 15 minutes. After removal of the crystal violet solution, biofilms were washed 3 times with water. For quantification of biofilm formation, dried stained biofilms were resuspended in $30 \%$ acetic acid and absorbance was measured at $585 \mathrm{~nm}$ using an infinite M200 PRO plate reader. Biofilm formation in different strains is represented in values normalized to average biofilm formation in the control strains.

\section{Competition assay for biofilm formation}

The glycerol stocks of the target strains were inoculated in LB medium supplemented with kanamycin $(20 \mu \mathrm{g} / \mathrm{mL})$ for over-day $6 \mathrm{~h}$ culture at $37^{\circ} \mathrm{C}$, then overnight in M63B1 minimum medium supplemented with $0.4 \%$ glucose and kanamycin. After overnight incubation, the cultures were adjusted to $\mathrm{OD}_{600}=1.0$ (ca. $5.0 \times 10^{8}$ bacteria $/ \mathrm{mL}$ ). The target strains with different fluorescent tags were mixed in 1:1 ratio and the cell concentrations were verified by FACS and OD analyses. Each competition mix was then inoculated in either a polystyrene Greiner 96-well plate or a polyvinyl chloride Corning plate at $\mathrm{OD}_{600}=0.01\left(5 \times 10^{6}\right.$ cells $\left./ \mathrm{mL}\right)$ in M63B1 minimum medium supplemented with $0.4 \%$ glucose and kanamycin. Incubation was done at $37^{\circ} \mathrm{C}$ overnight, for 16 hours. The supernatant was removed from each well and the biofilm was resuspended in $100 \mu \mathrm{L}$ of PBS. The proportion of each strain was then assessed from this resuspension by FACS and the relative fitness of target strain was calculated as follows:

$$
\text { relative fitness }=\frac{\left(\frac{\text { Target }_{\text {post }}}{\text { Target }_{\text {pre }}}\right)}{\left(\frac{\text { ref } f_{\text {post }}}{\text { ref } f_{\text {pre }}}\right)}
$$

, where Target pre $_{\text {and Target }}$ post are the cell concentrations of the target strain in the mixed culture before and after the overnight incubation, and $r e f_{\text {pre }}$ and $r e f_{\text {post }}$ are the cell concentrations of the reference strain in the mixed culture before and after the overnight incubation, respectively ${ }^{59}$.

\section{Competition assay for growth in liquid cultures}

The glycerol stocks of the target strains were inoculated in LB medium supplemented with kanamycin $(20 \mu \mathrm{g} / \mathrm{mL})$ for over-day $6 \mathrm{~h}$ culture, then for overnight in M63B1 minimum medium supplemented with $0.4 \%$ glucose and kanamycin. After overnight incubation, the 
cultures were adjusted to $\mathrm{OD}_{600}=1.0$ (ca. $5.0 \times 10^{8}$ bacteria $/ \mathrm{mL}$ ). The target strains with the different fluorescent tags were mixed in 1:1 ratio and the cell concentrations were verified by FACS and OD analyses. Then they were transferred into M63B1 minimum medium supplemented with $0.4 \%$ glucose and kanamycin at 1000 times dilution and incubated overnight. The concentrations of target and reference strains in the overnight culture was measured using FACS and spectrophotometer. The relative planktonic fitness during overnight culture were calculated as for competitions for biofilm formation assay (see above).

\section{Competition assay for initial adhesion}

515 Prior to the initial adhesion assay, the glycerol stocks of the target strains were inoculated in

516 culture tubes containing LB medium supplemented with kanamycin $(20 \mu \mathrm{g} / \mathrm{mL})$ for over-day 517 6h culture, then transferred into M63B1 minimum medium supplemented with $0.4 \%$ glucose 518 and kanamycin for overnight culture at $37^{\circ} \mathrm{C}$. The overnight culture were adjusted to $\mathrm{OD}_{600}=$ 5191.0 (ca. $5.0 \times 10^{8}$ bacteria $/ \mathrm{mL}$ ) and the target and reference strains with the different 520 fluorescent tag were mixed in 1:1 ratio. The ratio was verified by FACS analysis. Glass 521 spatulas were inoculated with $15 \mathrm{~mL}$ of the mixed culture for 10 mins and washed 3 times in $20 \mathrm{~mL}$ PBS. The bacteria attached to the glass spatula were resuspended in $10 \mathrm{~mL}$ minimum medium without glucose. The ratio of each strain in the resuspended solution was measured using FACS. The results were either expressed as (i) relative fitness for Figure S2 and were calculated as for competitions for biofilm formation assay (see above), or (ii) for Figure 4 as percent gain within the population as follows;

$$
(\text { percent gain within the population })[\%]=\left(\text { target }_{\text {adh }}-\text { target }_{\text {ino }}\right) \times \frac{50}{\text { target }_{\text {ino }}}
$$
, where the measured percentage of the target strain in the inoculation mixture and the resuspention are shown as $M T_{\text {ino }}$ and $M T_{a d h}$, respectively.

\section{RNA extraction and RT-PCR}

532 Three colonies of each strain were grown in LB medium supplemented with kanamycin (20 $\mu \mathrm{g} / \mathrm{mL}$ ) for over-day $6 \mathrm{~h}$ culture at $37^{\circ} \mathrm{C}$, then overnight in $25 \mathrm{~mL}$ of M63B1 minimum

534 medium supplemented with $0.4 \%$ glucose and kanamycin. RNA extractions were then 535 performed using Trizol Reagent (Ambion) following manufacturer instructions. Briefly, the 536 equivalent of $5.0 \times 10^{7}$ cells were centrifuged for 5 minutes at 5,000 rpm, the pellets were 537 resuspended in $1 \mathrm{~mL}$ of Trizol Reagent and incubated 5 minutes at room temperature. $200 \mu \mathrm{L}$ 538 of chloroform was added and each tube was vortexed for 15 seconds and incubated for 5 
minutes at room temperature before centrifugation for 15 minutes at $10,000 \mathrm{rpm}$ and at $4{ }^{\circ} \mathrm{C}$. The upper phase containing the total RNA was then transferred in $500 \mu \mathrm{L}$ of isopropanol. The tubes were mixed by inversion and incubated for 5 minutes at room temperature before centrifugation for 10 minutes at $10,000 \mathrm{rpm}$ and at $4^{\circ} \mathrm{C}$. The supernatant was discarded and

543 the pellets were washed by adding $1 \mathrm{~mL}$ of $70 \%$ ethanol. The tubes were mixed by inversion

544 and incubated for 5 minutes at room temperature before centrifugation for 10 minutes at $10,000 \mathrm{rpm}$ and at $4^{\circ} \mathrm{C}$. After removing the supernatant, the pellets were air dried and resuspended in $50 \mu \mathrm{L}$ of RNAse free water. A DNAse treatment was then applied to all samples using the TURBO DNAse kit (Ambion). The resulting RNA was used as a matrix for reverse transcription using the AMV cDNA first strand synthesis kit (Roche). The produced cDNA was used to assess ecpD expression as well as 16S rRNA gene as a control.

\section{Whole genome sequencing and analysis.}

552 Prior to genome extraction, the glycerol stocks of the target populations and clones were 553 inoculated in LB medium for over-day culture till the $\mathrm{OD}_{600}$ reached around 1.0 (ca. 5.0x $10^{8}$

554 bacteria/mL). The bacterial cells were collected from $2 \mathrm{~mL}$ of the culture and the genomic DNA was extracted using the Qiagen DNeasy Blood and Tissue kit. RNase digestion step was added during the genome extraction process. Sequencing libraries were prepared using Nextera XT or Nextera Flex DNA Library Preparation Kit. All samples were sequenced using Illumina HiSeq sequencer. Sequencing reads were pre-processed to remove low-quality or artefactual bases. We used fqCleaner v.0.5.0, a mini workflow implemented in Galaxy ${ }^{60}$ to

560 process fastq files (quality trimming, duplicate and artifact filters). Mutations with a

561 frequency superior to $5 \%$ were detected using breseq version $0.30 .0^{61}$ with the consensus mode for clones sequences analyses and the polymorphism mode for population sequencing.

563 In both cases default parameters were used.

\section{Determination of the frequency of fim $\mathrm{H}$ mutation}

566 The evolution of frequency of the identified mutations in fim $H$ was performed using Sanger

567 sequencing analysis of PCR products centered on the $\mathrm{fimH}$ region using the following

568 oligonucleotides - oligo up: AGGATGACAGTGGCAACACA and oligo down:

569 GTTTTGGCTTTTCGCACAAT. Small aliquots of the glycerol stocks corresponding to each 570 positive selection cycle were diluted in water and used for PCR reaction (Thermo Phusion 571 flash high-fidelity master mix). The PCR products were sent to Eurofins for purification and 
572 Sanger sequencing. The frequency of the mutations was calculated using QSVanalyzer ${ }^{62}$,

573 with a cutoff that does not allow detection of mutations lower than $5 \%$ frequency.

\section{Determination of fim "ON/OFF" status of evolved clones}

576 Orientation of the $314 \mathrm{bp}$ DNA segment harboring the fimA promoter (fimS region) was

577 determined using a PCR-based assay using restriction fragment length dimorphism arising

578 from the orientation-dependent location of a unique BstUI restriction site within the amplified

579 DNA. Briefly, the switch ( fimS) region was amplified from a sample of overnight cultures

580 with oligonucleotides OL4 (5' CCGTAACGCAGACTCATCCTC 3') and OL20 (5'

581 GAGTTTTAATTTTCATGCTGCTTTCC 3') to generate a 726 bp PCR product. DNA was

582 amplified with Taq polymerase (Invitrogen) using the following PCR conditions: denaturing

583 at $94^{\circ} \mathrm{C}$ for $5 \mathrm{~min}$, followed by 30 cycles $\left(94^{\circ} \mathrm{C}\right.$ for $1 \mathrm{~min}, 58^{\circ} \mathrm{C}$ for $1 \mathrm{~min}$ and $72^{\circ} \mathrm{C}$ for 1

$584 \mathrm{~min}$ ) and a final extension of $10 \mathrm{~min}$ at $72^{\circ} \mathrm{C}$. Samples were cooled at $8^{\circ} \mathrm{C}, 10$ units of BstUI

585 (Nex England Biolabs) were added to each reaction and incubation was conducted at $37^{\circ} \mathrm{C}$ for

$5863 \mathrm{~h}$. Digested PCR products were resolved on 2\% agarose gels. Using this assay, phase ON

587 populations of bacteria yielded two DNA fragments 433 and 293 bp in length, whereas phase

588 OFF populations yielded two fragments of 539 and $187 \mathrm{bp}$. Mixed populations contained a

589 mixture of all four fragments.

\section{Protein modelling and structure prediction}

592 The 3D structure of the lectin domain of MG1655 FimH presented corresponds to the pdb 593 model $1 \mathrm{KLF}^{63}$. The 3D structures of the mutant FimH proteins were predicted using the 594 PHYRE2 Protein Fold Recognition Server and visualized with the MacPymol Software ${ }^{64,65}$.

\section{Type 1 fimbriae extraction.}

597 Surface exposed type 1 fimbriae was isolated by heat shock extraction: a $5 \mathrm{~mL}$ culture of each

598 strain was grown in $\mathrm{LB}$ at $37^{\circ} \mathrm{C}$ for $16 \mathrm{~h}$ and $\mathrm{OD}_{600}=10.0$ equivalent of cell culture was 599 harvested by centrifugation. The harvested cells were washed with $0.9 \% \mathrm{NaCl}$, collected by 600 centrifugation and resuspended with $75 \mathrm{mM} \mathrm{NaCl}, 0.5 \mathrm{mM}$ Tris-HCl, $\mathrm{pH}$ 7.4. The samples 601 were incubated at $60^{\circ} \mathrm{C}$ for $20 \mathrm{~min}$, cooled on ice for $3 \mathrm{~min}$, centrifuged, and then the 602 detached adhesins present in the supernatant were precipitated with 10\% TCA overnight. The 603 proteins were collected by centrifugation $\left(20,000 \mathrm{~g}, 1 \mathrm{~h}, 4^{\circ} \mathrm{C}\right)$, then the acquired pellet was 604 washed with $75 \%$ acetone and the proteins were dissolved in HEPES $10 \mathrm{mM}$. 
606 Detection of FimA by Western blot with anti-FimA antibodies.

607 The heat extracted proteins from $\mathrm{OD}_{600}=2$ culture was suspended in 1X Laemmli buffer with

$608250 \mathrm{U}$ of benzonase nuclease (Sigma E0114) and incubated for 5 minutes at $95^{\circ} \mathrm{C}$. The

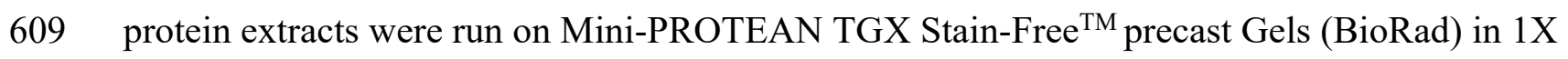

610 TGX buffer and then transferred to nitrocellulose membrane using a Trans-Blot ${ }^{\circledR}$ Turbo $^{\mathrm{TM}}$

611 Transfer System (BioRad). Blocking was performed in a 5\% solution of dry milk and 0.05\%

612 Tween $-1 \mathrm{X}$ PBS (1X PBST) overnight at $4{ }^{\circ} \mathrm{C}$ with agitation. The membranes were then

613 incubated in 1X PBST with a polyclonal rabbit antiserum raised against FimA subunit of type

6141 fimbriae (kindly given by Prof. Scott Hultgren) at 1:10000 for $1 \mathrm{~h}$ at room temperature with

615 agitation. Membranes were washed in 1X PBST and then incubated with the secondary

616 antibody (anti-rabbit IgG conjugated with horse radish peroxidase at 1:10000, Promega).

617 After washing the excess secondary antibody, specific bands were visualized using the ECL

618 prime detection method (GE Healthcare).

\section{Yeast agglutination assay}

621 The capacity of WT and E. coli mutants expressing FimH variant to bind yeast mannosylated proteins and agglutinate yeast cells was assessed as previously described ${ }^{66}$. The glycerol stocks of the PcL mutants and the PcL WT strains were inoculated in culture tubes containing

624 LB medium supplemented with kanamycin $(20 \mu \mathrm{g} / \mathrm{mL})$ for over-day $8 \mathrm{~h}$ culture, then

625 transferred into M63B1 minimum medium supplemented with $0.4 \%$ glucose and kanamycin

626 for overnight culture. The culture were washed once with $20 \mathrm{~mL}$ PBS and resuspended at

$627 \mathrm{OD}_{600}=9$ in PBS. $2 \% \mathrm{w} / \mathrm{v}$ yeast suspension was prepared using $100 \mathrm{mg}$ dry Saccharomyces cerevisiae (Sigma) and $5 \mathrm{~mL}$ PBS. A range of 12 two-fold dilutions of bacterial cells from OD600 $=9$ to OD600 $=0.02$ was tested for each strain. $50 \mu \mathrm{L}$ serial dilutions of bacteria in

630 PBS were mixed with $50 \mu \mathrm{L}$ yeast suspension in round-bottom 96 well-plates (Fisher). After

6313 hours of incubation (static condition) or 30 mins with $600 \mathrm{rpm}$ shaking (dynamic condition)

632 at room temperature, the lack of agglutination was seen as an aggregation of yeast cells at the

633 bottom of the well. In the presence of agglutination there was no yeast clumps but a

634 homogeneous mixture of yeast and bacteria. The agglutination titer corresponding to the

635 lowest concentration of bacteria leading to yeast agglutination was recorded for each strain.

\section{FimH sequences analysis}

638 The FimH sequence from the ancestral strain (E. coli K12, strain MG1655) used in the 639 positive selection experiment was used as a query for BLASTp searches (blast + version 
2.2.31, ${ }^{67}$ against the NCBI nrprot database (version 2019-04-09) as well as against a custom database composed of 2067 sequenced genomes of E. coli (supplementary Table S2) and 277 genomes from environmental strains published in ${ }^{26}$. Only hits corresponding to E.coli and with an e-value lower than $1 \mathrm{e}^{-10}$ and sequence identity higher than $70 \%$ were kept. In our analysis, the detection of one mutation in two identical sequences coming from different origin was important. Therefore, the redundancy of the FimH sequences dataset was not addressed in terms of sequence identity, but rather in term of isolates. To avoid such duplicated sequences, FimH proteins with identical identifiers or strain information were discarded. The resulting non-redundant dataset was composed of 3543 sequences (supplementary Table S3AB).

All sequences were aligned using mafft version $7.407{ }^{68}$ with G-INS-i option and the resulting alignment was screened to identify mutations relative to the reference sequence from $E$. coli K12, strain MG1655 using custom perl script. The same alignment was used to compute the single amino-acids modifications frequencies for each sequence in the signal peptide, mannose lectin domain, linker part and the pilin domain. For each sequence, the mutation frequency was computed for the whole sequence, the signal peptide, the lectin domain and the pilin domain. These dataset were then compared using a one-way Anova. To identify positions with a significantly higher mutation frequency, the same method was perfored using a sliding winow approach.

\section{Selection pressure analysis}

The $\omega$ ratio of non-synonymous $\left(\mathrm{d}_{\mathrm{N}}\right)$ to synonymous $\left(\mathrm{d}_{\mathrm{S}}\right)$ nucleotide substitutions is widely used to estimate the evolutionary forces acting on a gene ${ }^{69}$. A ratio of 1 is considered to indicate neutral evolution while a ratio higher than one indicates positive selection and a ratio lower than one indicates purifying selection. PAML version $4.9^{29}$ was used for the positive selection analyses. First, only unique sequences (100\% identity) were kept in the previous alignment (533 sequences), which was trimmed using trimal v1.4.1 ${ }^{70}$ and used as a guide to codon align the related nucleotide sequences using PAL2NAL version $14^{71}$. The resulting alignment was then used to compute a phylogenetic tree using PhyML version $3.1^{72}$ with 100 bootstraps and both were used as input for PAML. The best fit model for phylogenetic tree reconstruction was inferred using jModelTest2 version 2.1.3 ${ }^{73}$. The phylogenetic tree of Supp. Figure S8 was visualized and edited using iTOL v4 ${ }^{74}$. 
674 The site models M0, M3, M8a and M8 were first run on the whole tree. The comparison of

675 M0 vs M3 tests for rate heterogeneity between amino acid sites, while M8a vs M8 tests for

676 positive selection. These tests only detect positive selection acting on a site when the $\omega$ ratio

677 averaged over all branches is higher than one. However, positive selection could be restricted

678 to a sub-group of sequences, which is diluted in the whole tree. To identify such sub-groups

679 of FimH sequences subjected to positive selection we used two different strategies. (i) We

680 first divided the tree in four sub-groups ( $\mathrm{Sg} 1$ to $\mathrm{Sg} 4)$ based on visual inspection and

681 phylogroup distribution along the tree as well as positions 70 and 78 (91 and 99 in this study)

682 for which alleles $\mathrm{S}$ and $\mathrm{N}$, respectively, were described to be specific to UPEC, which allows

683 the discrimination of $\mathrm{Sg} 4$ and $\mathrm{Sg} 3$. The remaining sequences that were not assigned to $\mathrm{Sg} 1$ to

$684 \mathrm{Sg} 4$ were put in a fifth sub-group. Then we used the branch-site model A, which allows $\omega$ to

685 vary among sites in a specific branch of the tree (the foreground branch) ${ }^{75}$, by labeling $\operatorname{Sg} 1$ -

$686 \mathrm{Sg} 4$ as the foreground branch. Since $\mathrm{Sg} 5$ is not a monophyletic group, it was not investigated

687 using this method. (ii) The second strategy was to build new alignments and trees for each of

688 the five sub-groups and run models M0, M3, M8a and M8 on each of them separately.

690 Finally, to test if the lectin domain and the pilin domain evolved at different rates and were

691 subjected to different selective pressures, only these two domains were kept in the different

692 alignments (whole tree and sub-groups 1 to 5) and labeled as two different site partitions. Five

693 different fixed-site models were then run ${ }^{76}$ : model $\mathrm{A}$ assuming a single $\omega$ ratio for the entire

694 sequence; model B assuming different substitution rates; model $\mathrm{C}$ assuming different

695 substitution rates and codon frequencies; model D assuming different substitution rates,

696 different transition/transversion ratios and different $\omega$ ratios among the partitions; and model

697 D2 which is the same as model D but with a fixed transition/transversion ratio. The

698 comparison of model A vs model B tests for evolution of the two partitions at different rates.

699 The comparison of model B vs model C tests for different codon usage between the two

700 partitions. The comparison of model B vs model D tests for different transition/transversion

701 and $\omega$ ratios between both partitions. The last comparison between model B and model D2

702 allows to test for different $\omega$ ratios only.

704 In each case, a likelihood ratio test assuming a $\chi^{2}$ distribution was used to compare each pair

705 of models using twice the difference in log likelihoods as $\chi^{2}$ and the parameters difference

706 reported by PAML as degrees of freedom. The resulting $\mathrm{p}$-values were corrected in order to 
707 account for multiple testing and a FDR threshold of 0.05 was considered as significant. For

708 such statistical tests, posterior probabilities under Bayes Empirical Bayes ${ }^{77}$ analysis under

709 M8 model and branch-site model A were extracted to identify sites under positive selection.

710 To avoid local optima, all analyses were run with different starting $\omega$ values $(0.04,0.4$ and 4$)$.

711

\section{Statistical analysis}

713 Unpaired, non-parametric Mann-Whitney tests were performed using Prism 6.0 for Mac OS

$714 \mathrm{X}$ (GraphPad Software, Inc.) for CV staining biofilm assay in which each experiment was

715 performed at least 8 to 12 times. Unpaired t-test with Welch's corrections were performed in

716 the case of continuous flow biofilm experiments in which each experiment was performed 3

717 times.

718

719 


\section{DATA AVAILABILITY}

721 All sequencing reads were deposited in NCBI under the BioProject accession number PRJNA714528. The perl scripts used are available at https://github.com/Sthirietrupert/FimH_Evolution

\section{COMPETING FINANCIAL INTERESTS}

The authors declare no competing financial interests.

\section{ACKNOWLEDGEMENTS.}

730 We thank Rebecca Stevick, Médéric Diard and Olaya Rendueles for critical reading of the

731 manuscript. We are grateful to Prof. Scott Hultgren for kindly providing the anti-FimA

732 antiserum and to Dr. Olaya Rendueles for the initial help with the analysis of the mutations.

733 This work was supported by an Institut Pasteur grant, by the French government's

734 Investissement d'Avenir Program, Laboratoire d'Excellence "Integrative Biology of Emerging

735 Infectious Diseases" (grant $\mathrm{n}^{\circ}$ ANR-10-LABX-62-IBEID) and by the Fondation pour la

736 Recherche Médicale (grant DEQ20180339185). M.Y. was supported by Institut Pasteur Roux

737 Cantarini fellowship. S.T.-R was supported by the French National Research Agency (ANR),

738 project EvolTolAB (ANR-18-CE13-0010).

AUTHOR CONTRIBUTIONS: M.Y., C.B. and J.-M.G. designed the experiments. M.Y.,

742 L.M. and S.T.-R. performed the experiments. M.Y., C.B., S.T.-R., L.M. and J.-M.G. analyzed 743 data. J.-M.G, C.B., M.Y. and S.T.-R. wrote the paper. 


\section{REFERENCES}

$747 \quad 1 \quad$ Van Houdt, R. \& Michiels, C. W. Role of bacterial cell surface structures in

$748 \quad$ Escherichia coli biofilm formation. Res Microbiol 156, 626-633, doi:10.1016/j.resmic.2005.02.005 (2005).

7502 Beloin, C., Da Re, S. \& Ghigo, J. M. Colonization of Abiotic Surfaces. EcoSal Plus 1, doi:10.1128/ecosalplus.8.3.1.3 (2005).

753

754

755

756

3 Fronzes, R., Remaut, H. \& Waksman, G. Architectures and biogenesis doi:10.1038/emboj.2008.155 (2008).

$4 \quad$ Kline, K. A., Falker, S., Dahlberg, S., Normark, S. \& Henriques-Normark, B. Bacterial adhesins in host-microbe interactions. Cell Host Microbe 5, 580-592, doi:10.1016/j.chom.2009.05.011 (2009).

5 Patel, S., Mathivanan, N. \& Goyal, A. Bacterial adhesins, the pathogenic weapons to trick host defense arsenal. Biomed Pharmacother 93, 763-771, doi:10.1016/j.biopha.2017.06.102 (2017).

6 Korea, C. G., Badouraly, R., Prevost, M. C., Ghigo, J. M. \& Beloin, C. Escherichia coli K-12 possesses multiple cryptic but functional chaperone-usher fimbriae with distinct surface specificities. Environ Microbiol 12, 1957-1977, doi:10.1111/j.14622920.2010.02202.x (2010). potential role in environmental persistence. Environ Microbiol 18, 5228-5248, doi:10.1111/1462-2920.13559 (2016). Vo, J. L. et al. Autotransporter Adhesins
17, doi:10.1002/pmic.201600431 (2017).

9 Werneburg, G. T. \& Thanassi, D. G. Pili Assembled by the Chaperone/Usher Pathway in Escherichia coli and Salmonella. EcoSal Plus 8, doi:10.1128/ecosalplus.ESP-00072017 (2018).

10 Bhoite, S., van Gerven, N., Chapman, M. R. \& Remaut, H. Curli Biogenesis: Bacterial Amyloid Assembly by the Type VIII Secretion Pathway. EcoSal Plus 8, doi:10.1128/ecosalplus.ESP-0037-2018 (2019).

11 Beloin, C., Roux, A. \& Ghigo, J. M. Escherichia coli biofilms. Curr Top Microbiol Immunol 322, 249-289 (2008).

12 Valle, J. et al. Broad-spectrum biofilm inhibition by a secreted bacterial polysaccharide. Proc Natl Acad Sci US A 103, 12558-12563, doi:10.1073/pnas.0605399103 (2006).

13 Matthysse, A. G., Deora, R., Mishra, M. \& Torres, A. G. Polysaccharides cellulose, poly-beta-1,6-n-acetyl-D-glucosamine, and colanic acid are required for optimal binding of Escherichia coli O157:H7 strains to alfalfa sprouts and K-12 strains to plastic but not for binding to epithelial cells. Appl Environ Microbiol 74, 2384-2390, doi:10.1128/aem.01854-07 (2008).

14 Wang, X., Preston, J. F., 3rd \& Romeo, T. The pgaABCD locus of Escherichia coli promotes the synthesis of a polysaccharide adhesin required for biofilm formation. $J$ Bacteriol 186, 2724-2734, doi:10.1128/jb.186.9.2724-2734.2004 (2004).

15 Romling, U. \& Galperin, M. Y. Bacterial cellulose biosynthesis: diversity of operons, subunits, products, and functions. Trends Microbiol 23, 545-557, doi:10.1016/j.tim.2015.05.005 (2015). study gene function under physiological conditions: application to identification of 
801

802

803

804

805

806

807

808

809

810

811

812

813

814

815

816

817

818

819

820

821

822

823

824

825

826

827

828

829

830

831

832

833

834

835

836

837

838

839

840

841

842

new Escherichia coli adhesins. J Bacteriol 187, 1001-1013, doi:10.1128/JB.187.3.1001-1013.2005 (2005).

17 Wurpel, D. J., Beatson, S. A., Totsika, M., Petty, N. K. \& Schembri, M. A. Chaperone-usher fimbriae of Escherichia coli. PLoS One 8, e52835, doi:10.1371/journal.pone.0052835 (2013).

18 Chagnot, C., Zorgani, M. A., Astruc, T. \& Desvaux, M. Proteinaceous determinants of surface colonization in bacteria: bacterial adhesion and biofilm formation from a protein secretion perspective. Front Microbiol 4, 303, doi:10.3389/fmicb.2013.00303 (2013).

19 Chahales, P. \& Thanassi, D. G. Structure, Function, and Assembly of Adhesive Organelles by Uropathogenic Bacteria. Microbiol Spectr 3, doi:10.1128/microbiolspec.UTI-0018-2013 (2015).

20 Rossi, E. et al. "It's a gut feeling" - Escherichia coli biofilm formation in the gastrointestinal tract environment. Crit Rev Microbiol 44, 1-30, doi:10.1080/1040841x.2017.1303660 (2018).

21 Pratt, L. A. \& Kolter, R. Genetic analysis of Escherichia coli biofilm formation: roles of flagella, motility, chemotaxis and type I pili. Mol Microbiol 30, 285-293, doi:10.1046/j.1365-2958.1998.01061.x (1998).

22 Gally, D. L., Leathart, J. \& Blomfield, I. C. Interaction of FimB and FimE with the fim switch that controls the phase variation of type 1 fimbriae in Escherichia coli $\mathrm{K}$ 12. Mol Microbiol 21, 725-738, doi:10.1046/j.1365-2958.1996.311388.x (1996).

23 Klemm, P. Two regulatory fim genes, fimB and fimE, control the phase variation of type 1 fimbriae in Escherichia coli. Embo j 5, 1389-1393 (1986).

24 van der Woude, M. W. Phase variation: how to create and coordinate population diversity. Curr Opin Microbiol 14, 205-211, doi:10.1016/j.mib.2011.01.002 (2011).

25 Thomas, W. E., Trintchina, E., Forero, M., Vogel, V. \& Sokurenko, E. V. Bacterial adhesion to target cells enhanced by shear force. Cell 109, 913-923, doi:10.1016/s0092-8674(02)00796-1 (2002).

26 Touchon, M. et al. Phylogenetic background and habitat drive the genetic diversification of Escherichia coli. PLoS Genet 16, e1008866, doi:10.1371/journal.pgen.1008866 (2020).

27 Chen, S. L. et al. Positive selection identifies an in vivo role for FimH during urinary tract infection in addition to mannose binding. Proc Natl Acad Sci U S A 106, 2243922444, doi:10.1073/pnas.0902179106 (2009).

28 Weissman, S. J. et al. Differential stability and trade-off effects of pathoadaptive mutations in the Escherichia coli FimH adhesin. Infect Immun 75, 3548-3555, doi:10.1128/iai.01963-06 (2007).

29 Yang, Z. PAML 4: phylogenetic analysis by maximum likelihood. Mol Biol Evol 24, 1586-1591, doi:10.1093/molbev/msm088 (2007).

30 Korea, C. G., Ghigo, J. M. \& Beloin, C. The sweet connection: Solving the riddle of multiple sugar-binding fimbrial adhesins in Escherichia coli: Multiple E. coli fimbriae form a versatile arsenal of sugar-binding lectins potentially involved in surfacecolonisation and tissue tropism. Bioessays 33, 300-311, doi:10.1002/bies.201000121 (2011).

31 Lehti, T. A. et al. Phylogenetic group-associated differences in regulation of the common colonization factor Mat fimbria in Escherichia coli. Mol Microbiol 87, 12001222, doi:10.1111/mmi.12161 (2013).

32 Diderichsen, B. flu, a metastable gene controlling surface properties of Escherichia coli. J Bacteriol 141, 858-867 (1980). 
33 Henderson, I. R., Meehan, M. \& Owen, P. Antigen 43, a phase-variable bipartite outer

844

845

846

847

848

849

850

851

852

853

854

855

856

857

858

859

860

861

862

863

864

865

866

867

868

869

870

871

872

873

874

875

876

877

878

879

880

881

882

883

884

885

886

887

888

889

890

891

892

membrane protein, determines colony morphology and autoaggregation in Escherichia coli K-12. FEMS Microbiol Lett 149, 115-120, doi:10.1111/j.1574-

6968.1997.tb10317.x (1997).

34 Rodrigues, D. F. \& Elimelech, M. Role of type 1 fimbriae and mannose in the development of Escherichia coli K12 biofilm: from initial cell adhesion to biofilm formation. Biofouling 25, 401-411, doi:10.1080/08927010902833443 (2009).

35 Le Trong, I. et al. Structural basis for mechanical force regulation of the adhesin FimH via finger trap-like beta sheet twisting. Cell 141, 645-655, doi:10.1016/j.cell.2010.03.038 (2010).

36 Rosenberg, M. \& Kjelleberg, S. in Advances in microbial ecology p. 353-393. (Springer, 1986).

37 Ronald, L. S. et al. Adaptive mutations in the signal peptide of the type 1 fimbrial adhesin of uropathogenic Escherichia coli. Proc Natl Acad Sci U S A 105, 1093710942, doi:10.1073/pnas.0803158105 (2008).

38 Geng, J., Beloin, C., Ghigo, J. M. \& Henry, N. Bacteria hold their breath upon surface contact as shown in a strain of Escherichia coli, using dispersed surfaces and flow cytometry analysis. PLoS One 9, e102049, doi:10.1371/journal.pone.0102049 (2014).

39 Gbarah, A., Gahmberg, C. G., Ofek, I., Jacobi, U. \& Sharon, N. Identification of the leukocyte adhesion molecules CD11 and CD18 as receptors for type 1-fimbriated (mannose-specific) Escherichia coli. Infect Immun 59, 4524-4530 (1991).

40 Kukkonen, M. et al. Basement membrane carbohydrate as a target for bacterial adhesion: binding of type I fimbriae of Salmonella enterica and Escherichia coli to laminin. Mol Microbiol 7, 229-237, doi:10.1111/j.1365-2958.1993.tb01114.x (1993).

41 Ofek, I., Mirelman, D. \& Sharon, N. Adherence of Escherichia coli to human mucosal cells mediated by mannose receptors. Nature 265, 623-625, doi:10.1038/265623a0 (1977).

42 Sokurenko, E. V., Chesnokova, V., Doyle, R. J. \& Hasty, D. L. Diversity of the Escherichia coli type 1 fimbrial lectin. Differential binding to mannosides and uroepithelial cells. $J$ Biol Chem 272, 17880-17886, doi:10.1074/jbc.272.28.17880 (1997).

43 Sokurenko, E. V., Courtney, H. S., Ohman, D. E., Klemm, P. \& Hasty, D. L. FimH family of type 1 fimbrial adhesins: functional heterogeneity due to minor sequence variations among fimH genes. J Bacteriol 176, 748-755, doi:10.1128/jb.176.3.748755.1994 (1994).

44 Bouckaert, J. et al. The affinity of the FimH fimbrial adhesin is receptor-driven and quasi-independent of Escherichia coli pathotypes. Mol Microbiol 61, 1556-1568, doi:10.1111/j.1365-2958.2006.05352.x (2006).

45 Dreux, N. et al. Point mutations in FimH adhesin of Crohn's disease-associated adherent-invasive Escherichia coli enhance intestinal inflammatory response. PLoS Pathog 9, e1003141, doi:10.1371/journal.ppat.1003141 (2013).

46 Iebba, V. et al. Microevolution in fimH gene of mucosa-associated Escherichia coli strains isolated from pediatric patients with inflammatory bowel disease. Infect Immun 80, 1408-1417, doi:10.1128/iai.06181-11 (2012).

47 Stahlhut, S. G. et al. Comparative structure-function analysis of mannose-specific FimH adhesins from Klebsiella pneumoniae and Escherichia coli. J Bacteriol 191, 6592-6601, doi:10.1128/jb.00786-09 (2009).

48 Szunerits, S. et al. Differentiation of Crohn's Disease-Associated Isolates from Other Pathogenic Escherichia coli by Fimbrial Adhesion under Shear Force. Biology (Basel) 5, doi:10.3390/biology5020014 (2016). 
89349 Jang, J. et al. Environmental Escherichia coli: ecology and public health implicationsa review. J Appl Microbiol 123, 570-581, doi:10.1111/jam.13468 (2017).

50 Fabich, A. J. et al. Genotype and phenotypes of an intestine-adapted Escherichia coli K-12 mutant selected by animal passage for superior colonization. Infect Immun 79, 2430-2439, doi:10.1128/iai.01199-10 (2011).

51 Barroso-Batista, J. et al. The first steps of adaptation of Escherichia coli to the gut are dominated by soft sweeps. PLoS Genet 10, e1004182,

900 doi:10.1371/journal.pgen.1004182 (2014).

901 Ghalayini, M. et al. Evolution of a Dominant Natural Isolate of Escherichia coli in the Human Gut over the Course of a Year Suggests a Neutral Evolution with Reduced Effective Population Size. Appl Environ Microbiol 84, doi:10.1128/aem.02377-17 (2018).

53 Lescat, M. et al. Using long-term experimental evolution to uncover the patterns and determinants of molecular evolution of an Escherichia coli natural isolate in the streptomycin-treated mouse gut. Mol Ecol 26, 1802-1817, doi:10.1111/mec.13851 (2017).

54 Lourenço, M. et al. A Mutational Hotspot and Strong Selection Contribute to the Order of Mutations Selected for during Escherichia coli Adaptation to the Gut. PLoS Genet 12, e1006420, doi:10.1371/journal.pgen.1006420 (2016). Meric, G., Kemsley, E. K., Falush, D., Saggers, E. J. \& Lucchini, S. Phylogenetic

56 Bergholz, P. W., Noar, J. D. \& Buckley, D. H. Environmental patterns are imposed on the population structure of Escherichia coli after fecal deposition. Appl Environ Microbiol 77, 211-219, doi:10.1128/aem.01880-10 (2011). distribution of traits associated with plant colonization in Escherichia coli. Environ Microbiol 15, 487-501, doi:10.1111/j.1462-2920.2012.02852.x (2013).

\section{Ghigo, J. M. Natural conjugative plasmids induce bacterial biofilm development.} Nature 412, 442-445, doi:10.1038/35086581 (2001).

59 Lenski, R. E., Rose, M. R., Simpson, S. C. \& Tadler, S. C. Long-Term Experimental Evolution in Escherichia coli. I. Adaptation and Divergence During 2,000 Generations. The American Naturalist 138, 1315-1341, doi:10.1086/285289 (1991).

60 Afgan, E. et al. The Galaxy platform for accessible, reproducible and collaborative biomedical analyses: 2018 update. Nucleic Acids Res 46, W537-w544, doi:10.1093/nar/gky379 (2018).

61 Deatherage, D. E. \& Barrick, J. E. Identification of mutations in laboratory-evolved microbes from next-generation sequencing data using breseq. Methods Mol Biol 1151, 165-188, doi:10.1007/978-1-4939-0554-6_12 (2014).

62 Carr, I. M. et al. Inferring relative proportions of DNA variants from sequencing electropherograms. Bioinformatics 25, 3244-3250, doi:10.1093/bioinformatics/btp583 (2009).

63 Hung, C. S. et al. Structural basis of tropism of Escherichia coli to the bladder during urinary tract infection. Mol Microbiol 44, 903-915, doi:10.1046/j.13652958.2002.02915.x (2002).

64 Kelley, L. A., Mezulis, S., Yates, C. M., Wass, M. N. \& Sternberg, M. J. E. The Phyre2 web portal for protein modeling, prediction and analysis. Nature Protocols 10 , 845, doi:10.1038/nprot.2015.053 (2015).

65 PyMOL (PyMOL Molecular Graphics System, 2010). 
94266 Hasman, H., Schembri, M. A. \& Klemm, P. Antigen 43 and type 1 fimbriae determine

943 colony morphology of Escherichia coli K-12. J Bacteriol 182, 1089-1095,

944

945 doi:10.1128/jb.182.4.1089-1095.2000 (2000).

946

947

67 Camacho, C. et al. BLAST+: architecture and applications. BMC Bioinformatics 10, 421, doi:10.1186/1471-2105-10-421 (2009).

68 Yamada, K. D., Tomii, K. \& Katoh, K. Application of the MAFFT sequence alignment program to large data-reexamination of the usefulness of chained guide trees. Bioinformatics 32, 3246-3251, doi:10.1093/bioinformatics/btw412 (2016).

69 Yang, Z. \& Bielawski, J. P. Statistical methods for detecting molecular adaptation. Trends Ecol Evol 15, 496-503, doi:10.1016/s0169-5347(00)01994-7 (2000).

953

70 Capella-Gutierrez, S., Silla-Martinez, J. M. \& Gabaldon, T. trimAl: a tool for

954

955 automated alignment trimming in large-scale phylogenetic analyses. Bioinformatics 25, 1972-1973, doi:10.1093/bioinformatics/btp348 (2009).

71 Suyama, M., Torrents, D. \& Bork, P. PAL2NAL: robust conversion of protein

956

957

958

959

960

961

962

963

964

965

966

967

968 sequence alignments into the corresponding codon alignments. Nucleic Acids Res 34, W609-612, doi:10.1093/nar/gk1315 (2006).

72 Guindon, S. \& Gascuel, O. A simple, fast, and accurate algorithm to estimate large phylogenies by maximum likelihood. Syst Biol 52, 696-704, doi:10.1080/10635150390235520 (2003).

73 Darriba, D., Taboada, G. L., Doallo, R. \& Posada, D. jModelTest 2: more models, new heuristics and parallel computing. Nat Methods 9, 772, doi:10.1038/nmeth.2109 (2012).

74 Letunic, I. \& Bork, P. Interactive Tree Of Life (iTOL) v4: recent updates and new developments. Nucleic Acids Res 47, W256-w259, doi:10.1093/nar/gkz239 (2019).

75 Yang, Z. \& dos Reis, M. Statistical properties of the branch-site test of positive selection. Mol Biol Evol 28, 1217-1228, doi:10.1093/molbev/msq303 (2011).

76 Yang, Z. \& Swanson, W. J. Codon-substitution models to detect adaptive evolution that account for heterogeneous selective pressures among site classes. Mol Biol Evol 19, 49-57, doi:10.1093/oxfordjournals.molbev.a003981 (2002). Yang, Z., Wong, W. S. \& Nielsen, R. Bayes empirical bayes inference of amino acid sites under positive selection. Mol Biol Evol 22, 1107-1118, doi:10.1093/molbev/msi097 (2005). 


\section{FIGURES}

A

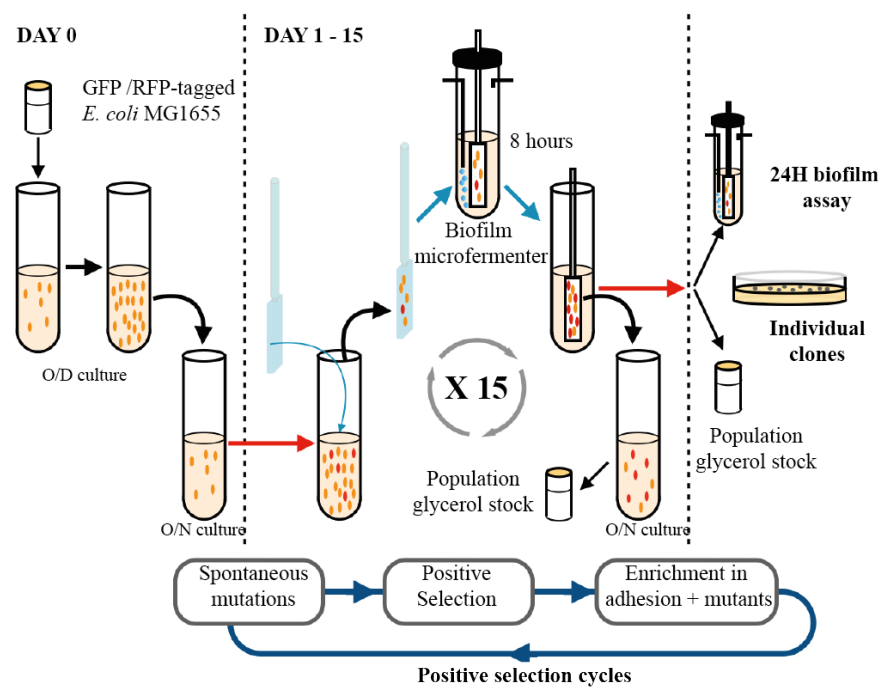

D

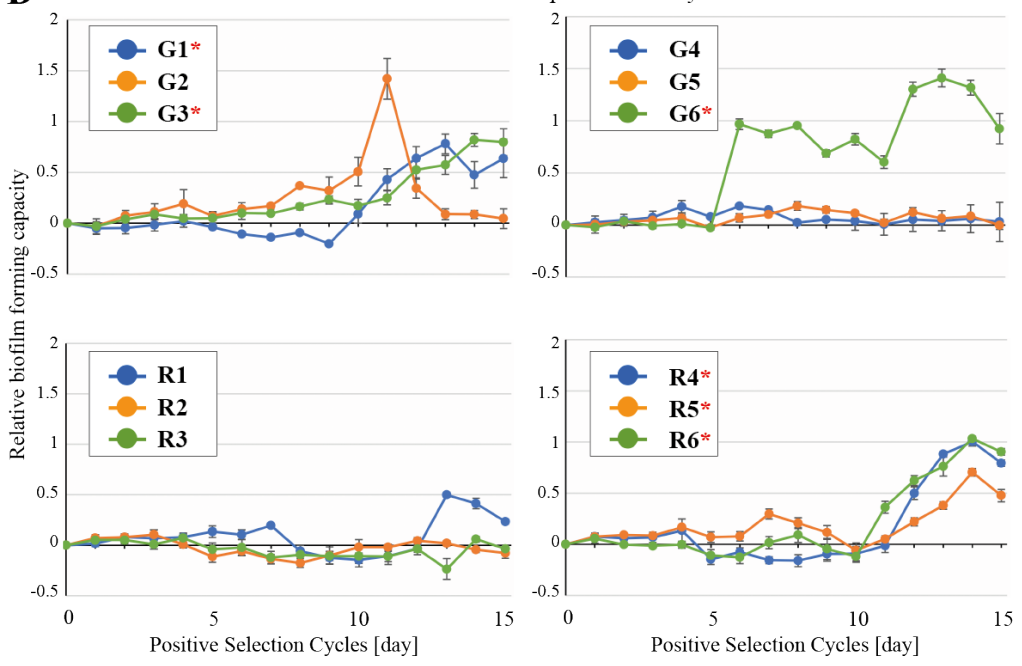

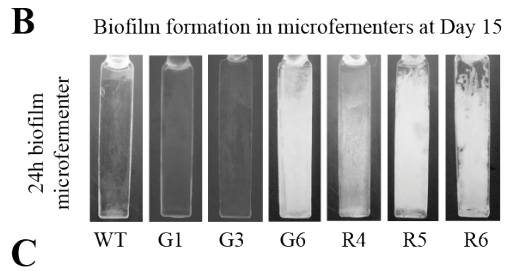

C
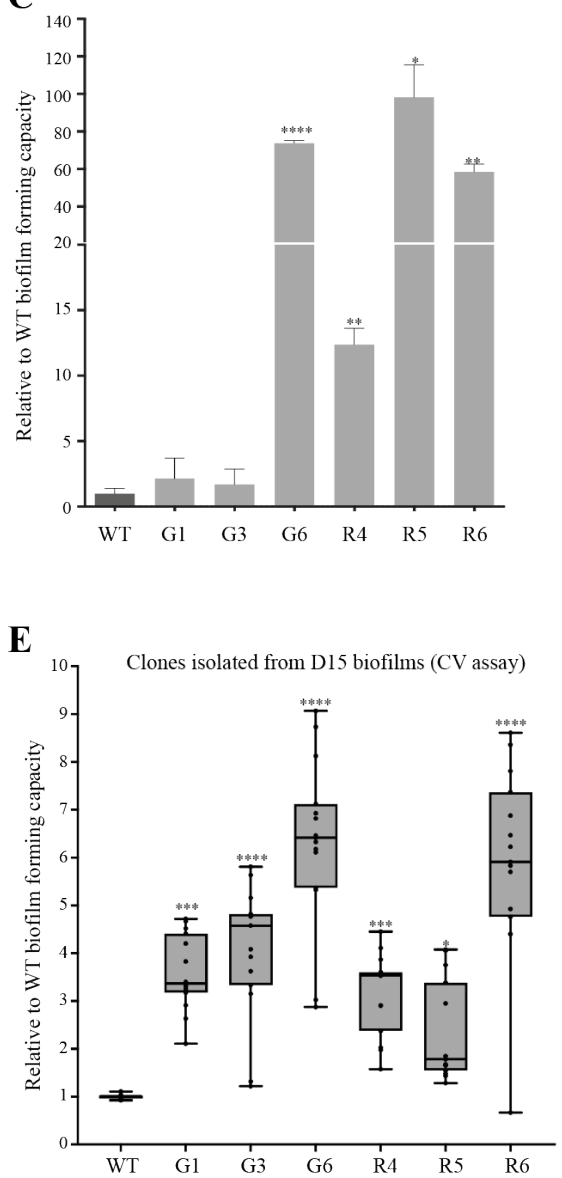

977

978

Figure 1. Selection of $E$. coli mutants with increased biofilm capacity

A. Schematics of positive selection to identify GFP- or RFP-tagged E. coli MG1655 mutants with increased biofilm formation capacity. B. Comparison of biofilm formation on biofilm microfermenters spatula of end-point populations. C. Comparison of biofilm biomass of endpoint populations and controls in biofilm microfermenters. The biofilm biomasses were collected from the spatula incubated for 24 hours and the biofilm-forming capacities were calculated relative to the one obtained with the control ancestral WT (set to 1). D. Evolution of biofilm forming capacity of bacterial population subjected to positive selection monitored by performing crystal violet biofilm assay using an aliquot of the population produced at each selection cycle. Comparison with the parental wild type strain and normalization were performed by calculating relative value using WT E. coli MG1655 (poor biofilm former) and 
990 E. coli $\mathrm{TG} 1$ strains as follows: $\left(\mathrm{OD}_{\mathrm{test}}-\mathrm{OD}_{\mathrm{WT}}\right) /\left(\mathrm{OD}_{\mathrm{TG} 1}-\mathrm{OD}_{\mathrm{WT}}\right)$. When the value is equal to 1

991 and 0, the capacity is as same as the TG1 strain and the WT MG1655 parental strain,

992 respectively. The experiments were performed in triplicate. G1-6 populations derive from

993 parental GFP-tagged MG1655. R1-6 populations derive from parental RFP-tagged MG1655.

994 The selected populations with increased biofilm capacity are indicated with an asterisk. E.

995 Comparison of biofilm forming capacities of 15 clones isolated from the selected population

996 at cycle 15 by crystal violet biofilm assay. Each point represents the data of an individual

997 clone. Each solid line in the boxplot shows the median of the biofilm forming capacities, and

998 the boxes illustrate the first and the third quartiles. The relative biofilm capacities were

999 calculated using the capacities of the parental wild type strain (median set to 1). All crystal

1000 violet biofilm assay experiments were conducted with at least 8 replicates. Continuous flow

1001 biofilm experiments in microfermenters were performed 3 times $(\mathbf{C})$ and statistics correspond

1002 to unpaired t-test with Welch's corrections. In $\mathbf{E}$ (12 répétitions) statistics correspond to

1003 unpaired, non-parametric Mann-Whitney test comparing all conditions to ancestral WT. *

$1004 \mathrm{p}<0.05 ; * * \mathrm{p}<0.01 ; * * * \mathrm{p}<0.001 ; * * * * \mathrm{p}<0.0001$

1005

1006 


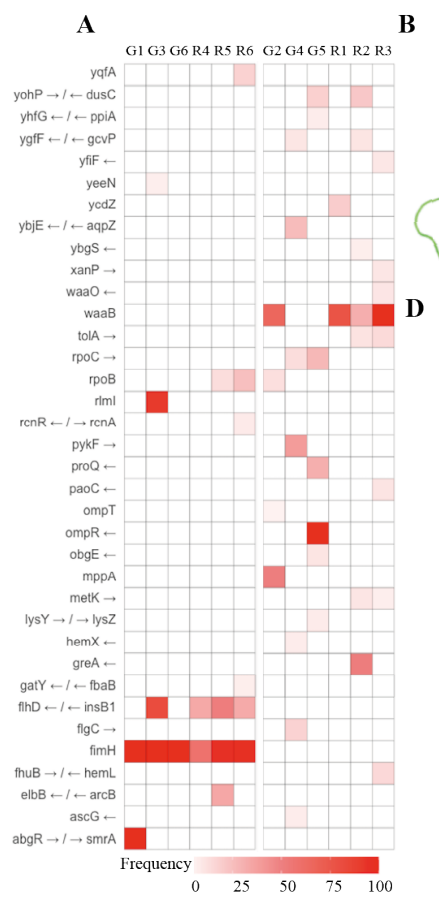

B
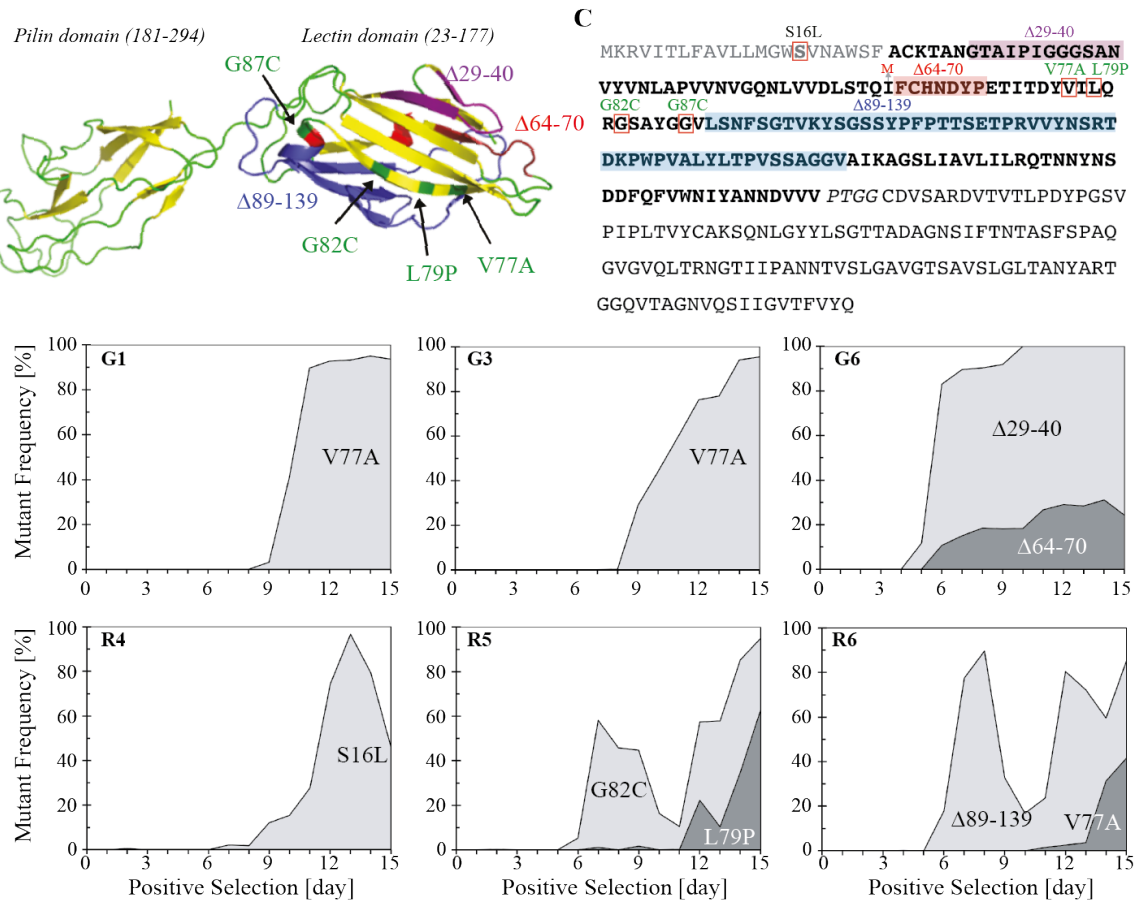

Figure 2. Nature and frequency of FimH mutations identified in evolved populations

with increased biofilm capacity. A. Frequency of mutation in targeted genes. Frequency of mutations inferred from population sequencing in the 6 populations with increased biofilm formation capacity (left) and the 6 populations without increased biofilm capacity (right). The frequency is color coded in red according to the legend at the bottom of the heatmap and correspond to the total frequency of all mutations in each gene within a population at cycle 15 locations of amino acid substitutions are indicated with black arrows and the in-frame deletions are indicated in purple $(\Delta 29-40)$, red $(\Delta 64-70)$ and blue $(\Delta 89-139)$. C. Identified FimH mutations are indicated above the FimH amino acid sequence. Single amino acid substitutions are highlighted with red boxes and three deletions are highlighted in pale purple, red and blue. The G87C mutation was identified in a distinct positive selection experiment using less selection cycles (4 instead of 15) and longer residence in the biofilm mutations acquired during the positive selection experiment in populations with increased adhesion capacity (G1, G3, G6, R4, R5, R6). The grey areas show the frequency of the mutants and the white area shows the frequency of the wild type strains. The frequencies were calculated using Sanger sequencing of fim $H$ region of sampled populations. 
A

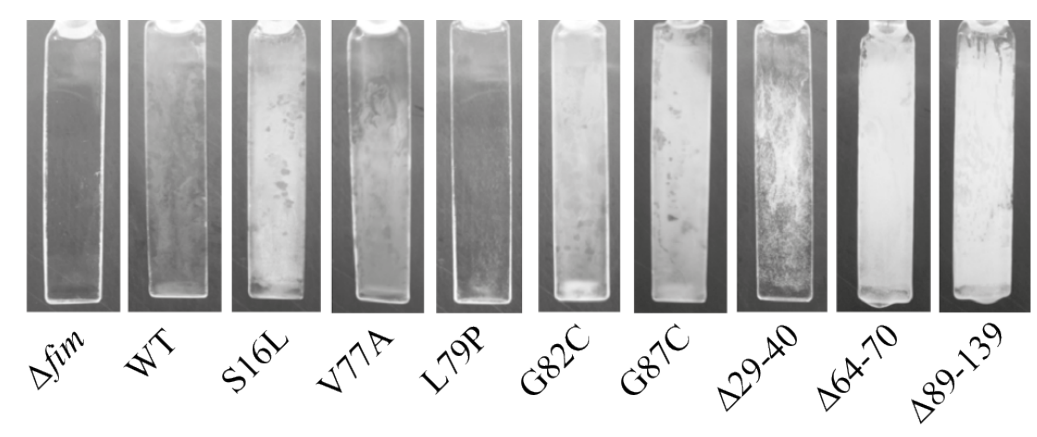

B

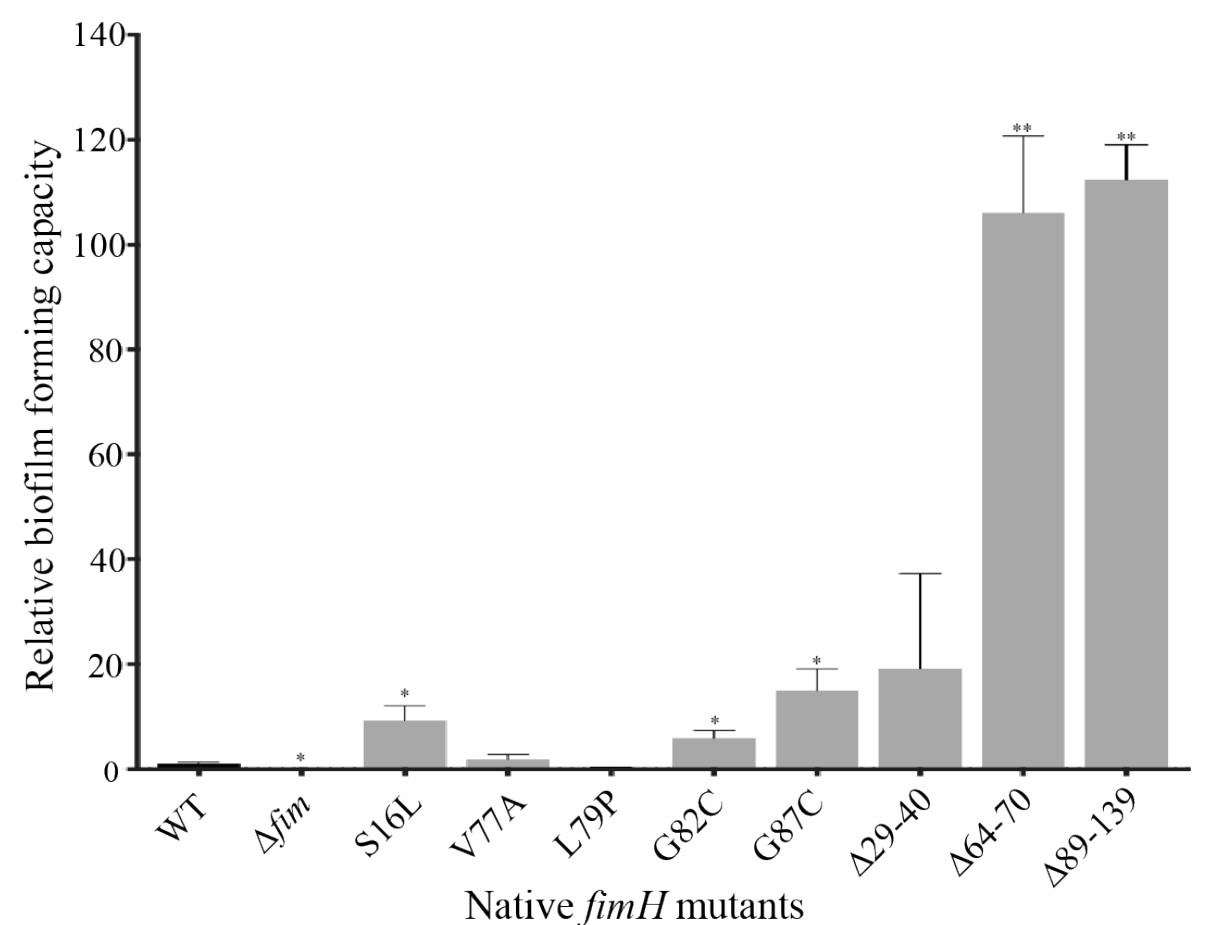

Figure 3. Biofilm formation capacity of individual fimH mutants. Biofilm formation of of representative biofilm biomass formed on biofilm microfermenters spatulas. B. The relative biofilm-forming capacities of individual fim $\mathrm{H}$ mutants was determined by comparison with the capacities of the $\Delta f i m(0)$ and wild type strains (1). Microfermenter biofilm experiments were performed in triplicate and statistics correspond to unpaired t-test with Welch's corrections.. ${ }^{*} \mathrm{p}<0.05 ; * * \mathrm{p}<0.01$. 

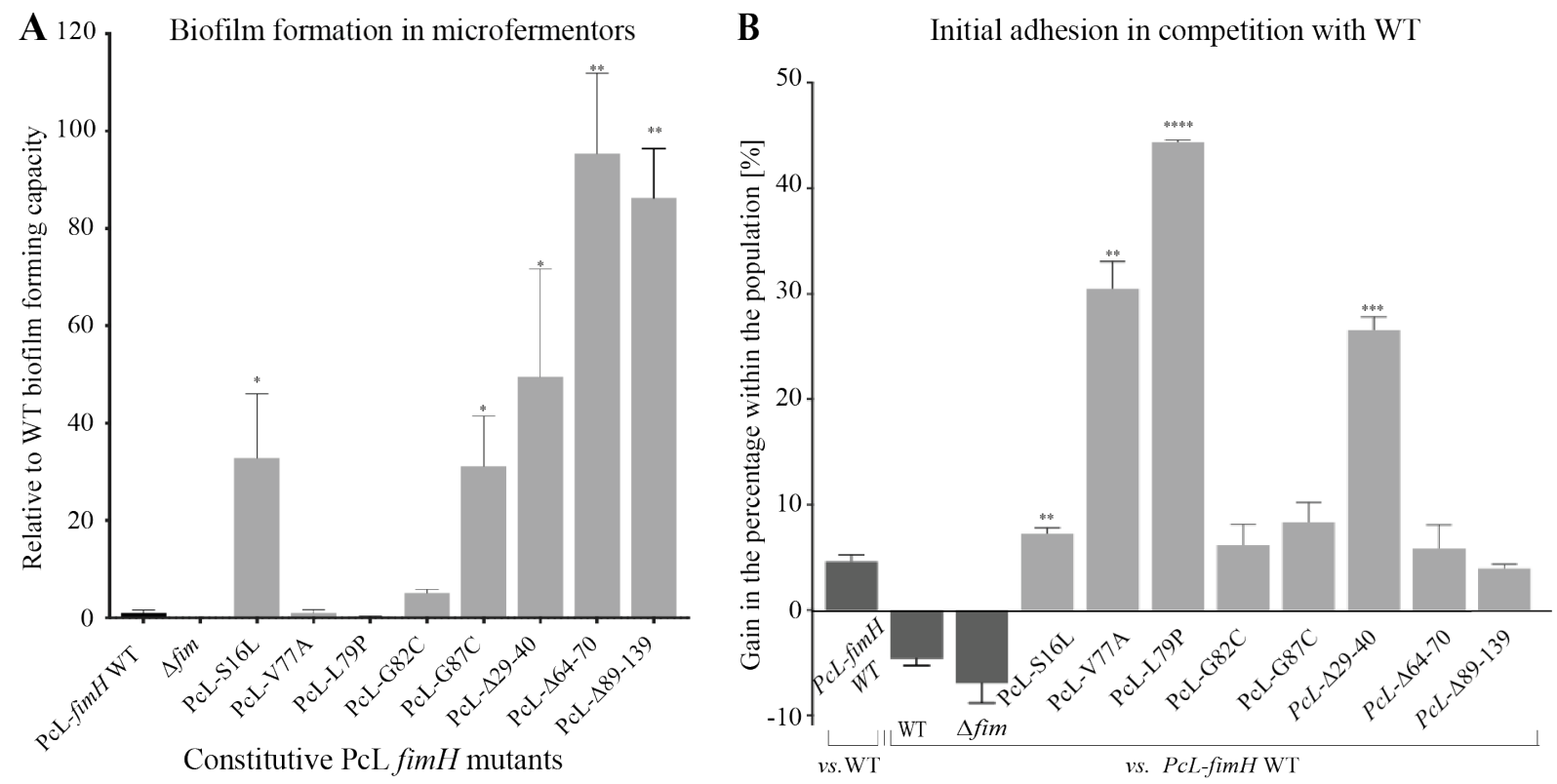

1044 Figure 4. Biofilm and initial adhesion capacity of constitutive PcL fimH mutants. A.

1045 Biofilm formation of $\Delta$ fim and PcLfimH WT strains (controls) and individual PcLfimH

1046 mutants after 24 hours incubation in biofilm microfermenters. The relative biofilm-forming capacities of individual PcL fimH mutants was determined by comparison with the capacities of the $\Delta$ fim (0) and PcLfimH WT strains (1). All biofilm experiments were performed in triplicate. B. Initial adhesion of PcLfimH mutants in competition with PcLfimH WT. The PcLfim $H$ mutants were inoculated to the microfermenter spatula in 1:1 ratio with PcLfimH WT strain carrying either GFP or RFP fluorescent tags for $10 \mathrm{~min}$, followed by washing, resuspension of the attached bacteria and CFU counting. The changes in the percentage of each strain within the population are shown. PcLfimH WT is also compared with the other control strains (WT and $\Delta$ fim strains, and PcLfimH WT strain with the different fluorescent tag). Statistics correspond to unpaired t-test with Welch's corrections. comparing all conditions to ancestral WT. ${ }^{*} \mathrm{p}<0.05 ; * * \mathrm{p}<0.01 ; * * * \mathrm{p}<0.001 ; * * * * \mathrm{p}<0.0001$. 


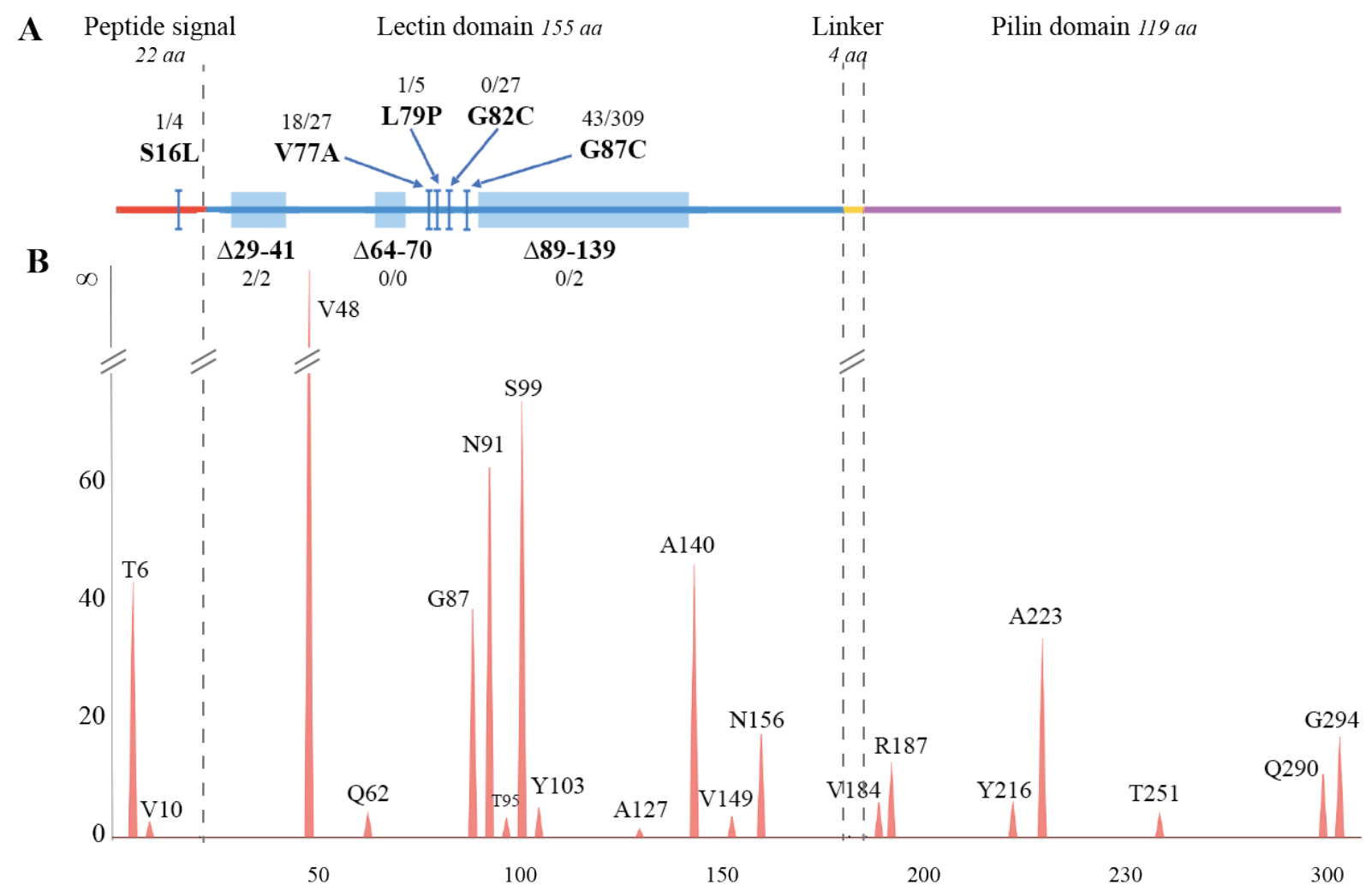

Figure 5. Analysis of the mutational spectrum of FimH sequences. A. Linear structure and amino-acid size of FimH protein domains with the eight mutations selected during the experimental evolution. The two numbers provided for each mutation correspond to respectively, the number of mutations among the 3266 analyzed FimH sequences and the number of sequences having a mutation at the same position in this study (regardless of mutation type). B. Negative log-transformed significance (-log10(pvalue)) of the mutation

1067 frequency at each position of the sequence relatively to the frequency calculated over all sequences from Database sequences. A one-way Anova was performed, the resulting p-values were adjusted and a False Discovery Rate (FDR) of 0.05 was considered as significant. Only positions with a significantly higher mutation frequency are plotted. 
$\mathbf{A}$
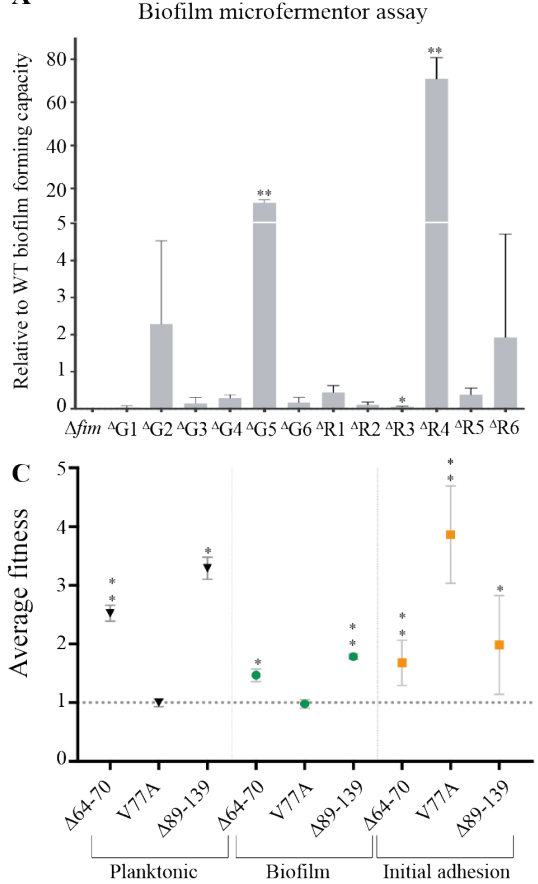

B
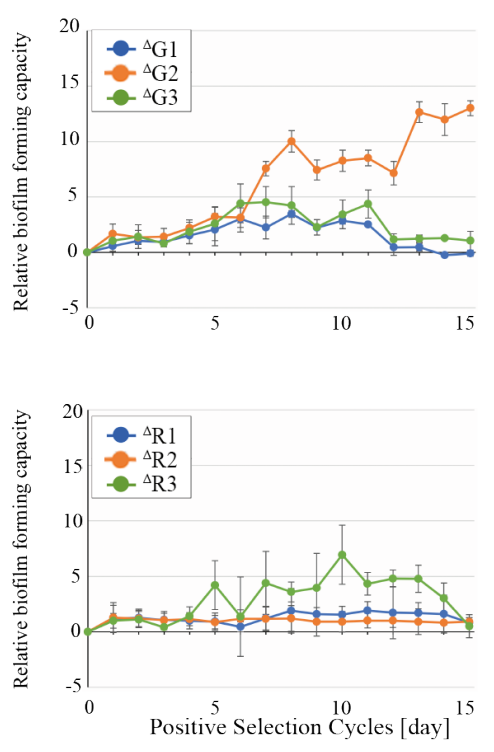

Biofilm microtiter plate CV assay
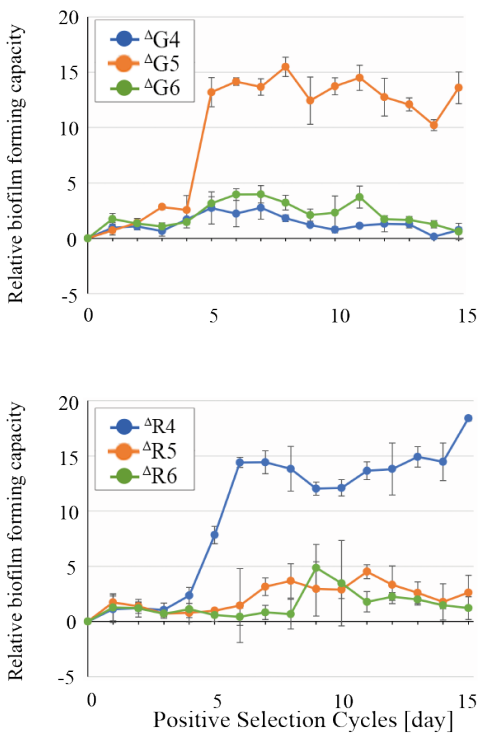

Figure 6. Evolution of biofilm forming capacity of $\Delta$ fim bacterial populations subjected

to positive selection for adhesion. A. The relative biofilm forming capacities of the populations at the end of the ${ }^{\Delta \text { fim }}$ positive selection experiment, after 24 hours in microfermenters. The relative capacities are calculated using the capacities of the wild type (1) and $\Delta$ fim ancestral strains (0). All microfermenter biofilm experiments were performed in monitored at each cycle during the ${ }^{\Delta \mathrm{fim}}$ positive selection experiment with the crystal violet assay of an aliquot of the enriched population as well as the parental wild type strains. The relative capacities were calculated using WT E. coli MG1655 $\Delta$ fim (very poor biofilm former) and E. coli $\mathrm{MG} 1655$ strain as follows: $\left(\mathrm{OD}_{\text {test }}-\mathrm{OD}_{\Delta \mathrm{fim}}\right) /\left(\mathrm{OD}_{\mathrm{MG} 1655}-\mathrm{OD}_{\Delta \mathrm{fim}}\right)$.. When the value is equal to 1 and 0 , the capacity is as same as the WT parental and the $\Delta$ fim strains, respectively. Each panel shows three populations: ${ }^{\Delta \mathrm{fim}} \mathrm{G} 1-3,{ }^{\Delta \mathrm{fim}} \mathrm{G} 4-6,{ }^{\Delta \mathrm{fim}} \mathrm{R} 1-3$ and ${ }^{\Delta \mathrm{fim}} \mathrm{R} 4-6$. All crystal violet biofilm assay experiments were conducted with at least 8 replicates. C. Comparison of fitness for three fim $\mathrm{H}$ mutants under native promoter competed against the best biofilm former mutant from the $\Delta$ fim evolution experiment (ecpR IS2(-) $+5 \mathrm{bp} /$ ecpD G89D). The strains were competed for planktonic growth, biofilm formation and initial adhesion on the microfermentor glass spatula as described in Materials and Methods. Dotted line represents ecpD/ecpR mutant fitness set to 1 . Statistics correspond to unpaired, non-parametric MannWhitney test comparing all conditions to WT $\Delta$ fim. ${ }^{*} \mathrm{p}<0.05 ;{ }^{* *} \mathrm{p}<0.01$. 


\section{SUPPLEMENTARY MATERIAL}

1098

1099

1100

1101

1102

1103

1104

1105

1106

1107

1108

1109

1110

1111

1112

1113

1114

\section{SUPPLEMENTARY TABLES}

Supplementary Table S1(available https://research.pasteur.fr/en/project/supplementary-tablesfor-biorxiv/): Mutations identified in populations and clones from the evolution in a WT genetic background at cycle 15 of the experiments. Datasheet $A$ in the 6 biofilm positive populations, Datasheet B in the 6 biofilm negative populations.

For each mutation identified the gene, nature of the mutation, impact on the protein sequence as well as the frequency of the mutation in the population are shown.

Supplementary Table S2 (available https://research.pasteur.fr/en/project/supplementarytables-for-biorxiv/): $\boldsymbol{E}$. coli genomes used in this study

For each genome, the strain's NCBI taxonomy identifier is shown in the first column, the name of the corresponding strain in the second column and the related NCBI genome assembly identifier in the third column.

Supplementary Table S3 (available https://research.pasteur.fr/en/project/supplementarytables-for-biorxiv/): Summary table of all FimH sequences used in this study.For each sequence, the effective length is specified for the signal sequence, the mannose lectin domain, the linker part and the pilin domain as well as for the whole protein. The number of mutations and the related mutation frequencies (number of mutations per amino acid) are also specified. Features obtained from NCBI (Strain name, serotype, isolation source and host) are noted when available. The phylogroup was determined using ClermonTyping method (Beghain et al., 2018) and positions 70 and 78 (numbered 91 and 99 in this study) that were described as related to UPEC strains are also specified. Finally, sequences having a mutation at the same position than the eight mutations (five single amino acid changes and three in-frame deletions) selected during the experimental evolution are specified. The number of exact same mutation/the total number of mutations at this specific position are specified in cells $\mathrm{X} 4$ to AE4.

Supplementary Table S4 (available https://research.pasteur.fr/en/project/supplementarytables-for-biorxiv/): Phylogroups, strain origin and enrichment in the mutations found at the 20 hotspots.A. Phylogroup enrichment in the 361 sequences mutated at the same position than the mutants selected in our experiments as well as the 64 sequences having the exact 
1132

1133

1134

1135

1136

1137

1138

1139

1140

1141

1142

1143

1144

1145

1146

1148

1149

1150

1151

1152

1153

1154

1155

1156

1157

1158

1159

1160

1161

1162

1163

1164

1165

same mutations relatively to all FimH sequences from GenBank. Ns: not significant. B. The 20 hotspots identified in E. coli FimH sequences. For each hotspot the position is specified in the third column and the first column specifies the location in the sequence. All identified alleles are listed in the fourth column with the number of sequences bearing it in the following one. The last column shows the phylogroup enrichment related to each allele when applicable. C. Phylogroup enrichment in the sequences bearing co-occurring mutations. The percentage of each phylogroup is specified in each case with the associated p-value between parentheses (Fisher exact test). Values corresponding to enriched phylogroups are shown in red whereas depleted ones are in blue. ns = not significant. D. Phylogroups enrichments in tree sub-groups. For each sub-group, an exact test of Fisher was used to identify the enriched and depleted phylogroups relatively to the reminder of the tree. The p-values were FDR corrected and a threshold of 0.05 was considered as significant.

\section{Supplementary Table S5. (available https://research.pasteur.fr/en/project/supplementary-} tables-for-biorxiv/): Fixed-site models testing for different evolutionary pressures on the mannose lectin domain and the pilin domain.A. Comparison between M0 and M3 models testing for selection variability among sites and M8a and M8 models testing for positive selection. $\operatorname{lnL}$ is the log-likelihood value for each model. $\omega 0, \omega 1$ and $\omega 2$ are the $\mathrm{dN} / \mathrm{dS}$ ratios of the three site classes inferred by the M3 model and p0, p1 and p2 are the related proportions of sites in the sequence belonging to these site classes. P0, P1, P and q are the parameters estimated by the M8a and M8 models. For the BEB analysis, sites predicted to be under positive selection at the $99 \%$ level are in bold, the other are predicted at the $95 \%$ level.

B. Branch-site model A test for episodic positive selection in sub-groups 1 to 4 . $\operatorname{lnL}$ is the loglikelihood value for each model. In each case, the foreground branch is the sub-group under investigation and the background is the reminder of the tree. For the BEB analysis, sites predicted to be under positive selection at the $99 \%$ level are in bold, the other are predicted at the 95\% level. C. Fixed-site models testing for different evolutionary pressures on the mannose lectin domain and the pilin domain. The first partition corresponds to the lectin domain and the second to the pilin domain. $\mathrm{r} 2$ is the relative substitution rate of the second partition relatively to the first partition. $\mathrm{K} 1$ and $\mathrm{K} 2$ are the transition/transversion ratio for the first and the second partition, respectively. $\omega 1$ and $\omega 2$ are the $\mathrm{dN} / \mathrm{dS}$ ratio for the first and the second partition, respectively. D. Likelihood Ratio Test for fixed-sites models. Model A vs B tests for different substitution rates. Model B vs C tests for different substitution rates and codon frequencies. Model B vs D tests for different $\mathrm{K}$ and $\omega$ ratios. Model B vs D2 tests for 
1166 different $\omega$ ratios. The resulting p-values were FDR corrected and a threshold of 0.05 was

1167 considered as significant.

1168

1169 Supplementary Table S6 (available https://research.pasteur.fr/en/project/supplementary-

1170 tables-for-biorxiv//): Mutations identified in biofilm positives populations and

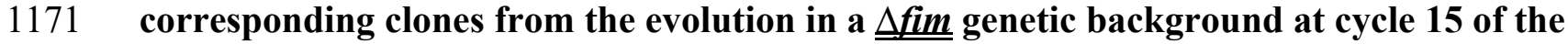

1172 experiments. SheetA mutations in the 3 biofilm positive populations, SheetB mutations in

1173 corresponding clones of the 3 biofilm positive populations.

1174 For each mutation identified the gene, nature of the mutation, impact on the protein sequence

1175 as well as the frequency of the mutation in the population are shown.

1176 
1177 Supplementary Table S7: E. coli strains used in this study

\begin{tabular}{|c|c|c|c|}
\hline Strain & Genotype-Phenotype & Relevant Information & Source \\
\hline MG1655 & $\mathrm{F}^{-}$lambda- $i l v G r f b-50 r p h-1$ & E. coli Genetic Stock Center GCSC\#6300 & $\begin{array}{l}\text { Laboratory } \\
\text { collection }\end{array}$ \\
\hline TG1 & $\begin{array}{l}\mathrm{F}^{\prime}\left[\text { traD36 proAB + lacl }{ }^{\mathrm{q}} \text { lacZDM15] }\right. \\
\text { supE hsdD5 thi } \Delta(\text { lac-proAB })\end{array}$ & Strong biofilm former & $\begin{array}{l}\text { Laboratory } \\
\text { collection }\end{array}$ \\
\hline WT $g f p$ & MG1655gfpmut3, $\mathrm{Km}^{\mathrm{R}}$ & $\begin{array}{l}\text { gfpmut } 3 \text { gene inserted at the } \lambda \text { att site under } \lambda \mathrm{P}_{\mathrm{R}} \\
\text { constitutive promoter }\end{array}$ & This study \\
\hline WT $r f p$ & MG1655mars, $\mathrm{Km}^{\mathrm{R}}$ & $\begin{array}{l}\text { mars rfp gene inserted at the } \lambda \text { att site under } \lambda \mathrm{P}_{\mathrm{R}} \\
\text { constitutive promoter }\end{array}$ & This study \\
\hline PcLfimH WT & $\begin{array}{l}\text { MG1655gfpmut3_specPcLfim, } \\
\mathrm{Km}^{\mathrm{R}}, \mathrm{Spec}^{\mathrm{R}}\end{array}$ & WT with constitutive type 1 fimbriae & This study \\
\hline$\Delta f i m g f p$ & $\begin{array}{l}\text { MG1655gfpmut3_tfim::cat, } \\
\mathrm{Km}^{\mathrm{R}}, \mathrm{Cm}^{\mathrm{R}}\end{array}$ & WT with deleted type 1 fimbriae & Korea e tal (1). \\
\hline$\Delta$ fim $r f p$ & $\begin{array}{l}\text { MG1655mars_tfim::cat, } \\
\mathrm{Km}^{\mathrm{R}}, \mathrm{Cm}^{\mathrm{R}}\end{array}$ & WT with deleted type 1 fimbriae & Korea e tal (1). \\
\hline S16L & $\begin{array}{l}\text { MG1655mars_fimH_S16L, } \\
\mathrm{Km}^{\mathrm{R}}\end{array}$ & $\begin{array}{l}\text { WT with S16L mutation in fimH, isolated from } \\
\text { population R4D15 }\end{array}$ & This study \\
\hline V77A & $\begin{array}{l}\text { MG1655mars_fimH_V77A, } \\
\mathrm{Km}^{\mathrm{R}}\end{array}$ & $\begin{array}{l}\text { WT with V77A mutation in fimH, isolated from } \\
\text { population R6D15 }\end{array}$ & This study \\
\hline L79P & $\begin{array}{l}\text { MG1655mars_fimH_L79P, } \\
\mathrm{Km}^{\mathrm{R}}\end{array}$ & $\begin{array}{l}\text { WT with L79P mutation in fimH, isolated from } \\
\text { population R5D15 }\end{array}$ & This study \\
\hline G82C & $\begin{array}{l}\text { MG1655mars_fimH_G82C, } \\
\mathrm{Km}^{\mathrm{R}}\end{array}$ & $\begin{array}{l}\text { WT with G82C mutation in fim H, isolated from } \\
\text { population R5D15 }\end{array}$ & This study \\
\hline G87C & $\begin{array}{l}\text { MG1655gfpmut3_fimH_G87C } \\
, \mathrm{Km}^{\mathrm{R}}\end{array}$ & $\begin{array}{l}\text { WT with G87C mutation in fim H, isolated from } \\
\text { population PS2G4 }\end{array}$ & This study \\
\hline$\Delta 29-40$ & $\begin{array}{l}\text { MG1655gfpmut3_fimH_d29- } \\
40, \mathrm{Km}^{\mathrm{R}}\end{array}$ & $\begin{array}{l}\text { WT with } \triangle 29-40 \text { mutation in fim } H \text {, isolated from } \\
\text { population G6D15 }\end{array}$ & This study \\
\hline$\Delta 64-70$ & $\begin{array}{l}\text { MG1655gfpmut3_fimH_d64- } \\
70, \mathrm{Km}^{\mathrm{R}}\end{array}$ & $\begin{array}{l}\text { WT with } \triangle 64-70 \text { mutation in fim } H \text {, isolated from } \\
\text { population G6D15 }\end{array}$ & This study \\
\hline$\Delta 89-139$ & $\begin{array}{l}\text { MG1655mars_fimH_ } \Delta 89-139 \text {, } \\
\mathrm{Km}^{\mathrm{R}}\end{array}$ & $\begin{array}{l}\text { WT with } \Delta 89-139 \text { mutation in } \text { fimH, isolated from } \\
\text { population R6D15 }\end{array}$ & This study \\
\hline PcL-S16L & $\begin{array}{l}\text { MG1655mars_specPcLfim } \\
\text { fimH_S16L, } \mathrm{Km}^{\mathrm{R}}, \mathrm{Spec}^{\mathrm{R}}\end{array}$ & $\begin{array}{l}\text { S16L mutation in fimH and constitutive type } 1 \\
\text { fimbriae }\end{array}$ & This study \\
\hline PcL-V77A & $\begin{array}{l}\text { MG1655mars_specPcLfim } \\
\text { fimH_V77A, } \mathrm{Km}^{\mathrm{R}}, \mathrm{Spec}^{\mathrm{R}}\end{array}$ & $\begin{array}{l}\text { V77A mutation in fimH and constitutive type } 1 \\
\text { fimbriae }\end{array}$ & This study \\
\hline PcL-L79P & $\begin{array}{l}\text { MG1655mars_specPcLfim } \\
\text { fimH_L79P, } \mathrm{Km}^{\mathrm{R}}, \mathrm{Spec}^{\mathrm{R}}\end{array}$ & $\begin{array}{l}\text { L79P mutation in fimH and constitutive type } 1 \\
\text { fimbriae }\end{array}$ & This study \\
\hline PcL-G82C & $\begin{array}{l}\text { MG1655mars_specPcLfim } \\
\text { fimH_G82C, } \mathrm{K} m^{\mathrm{R}}, \text { Spec }^{\mathrm{R}}\end{array}$ & $\begin{array}{l}\text { G82C mutation in fimH and constitutive type } 1 \\
\text { fimbriae }\end{array}$ & This study \\
\hline PcL-G87C & $\begin{array}{l}\text { MG1655gfpmut3_specPcLfim } \\
\text { fimH_G87C, } \mathrm{Km}^{\mathrm{R}}, \mathrm{Spec}^{\mathrm{R}}\end{array}$ & $\begin{array}{l}\text { G87C mutation in fimH and constitutive type } 1 \\
\text { fimbriae }\end{array}$ & This study \\
\hline PcL- $\Delta 29-40$ & $\begin{array}{l}\text { MG1655gfpmut3_specPcLfim } \\
\text { fimH_d29-40, } \mathrm{Km}^{\mathrm{R}}, \mathrm{Spec}^{\mathrm{R}}\end{array}$ & $\begin{array}{l}\Delta 29-40 \text { mutation in fim } H \text { and constitutive type } 1 \\
\text { fimbriae }\end{array}$ & This study \\
\hline PcL- $\Delta 64-70$ & $\begin{array}{l}\text { MG1655gfpmut3_specPcLfim } \\
\text { fimH_d64-70, } \mathrm{Km}^{\mathrm{R}}, \mathrm{Spec}^{\mathrm{R}}\end{array}$ & $\begin{array}{l}\Delta 64-70 \text { mutation in fim } H \text { and constitutive type } 1 \\
\text { fimbriae }\end{array}$ & This study \\
\hline PcL- $\Delta 89-139$ & $\begin{array}{l}\text { MG1655mars_specPcLfim } \\
\text { fimH_ } \Delta 89-139, \mathrm{Km}^{\mathrm{R}}, \mathrm{Spec}^{\mathrm{R}}\end{array}$ & $\begin{array}{l}\Delta 89-139 \text { mutation in } \text { fim } H \text { and constitutive type } 1 \\
\text { fimbriae }\end{array}$ & This study \\
\hline ecpR & 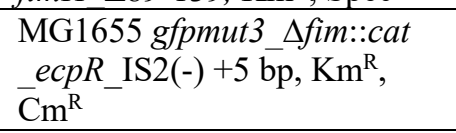 & $\Delta$ fim with the IS insertion right after ecpR & This study \\
\hline ecpD/ecpR & $\begin{array}{l}\text { MG1655 mars } \\
\Delta \text { _fim::cat_ecpD_G89D_ecpR } \\
\text { IS2(-) }+5 \mathrm{bp}, \mathrm{Km}^{\mathrm{R}}, \mathrm{Cm}^{\mathrm{R}}\end{array}$ & $\begin{array}{l}\Delta f i m \text { with G89R mutation in } e c p D \text { and the IS } \\
\text { insertion right after } e c p R\end{array}$ & This study \\
\hline
\end{tabular}


1180 Supplementary Table S8: List of primers used in this study

\begin{tabular}{|c|c|}
\hline \multicolumn{2}{|c|}{ fimH Sanger sequencing } \\
\hline fimH-F & AGGATGACAGTGGCAACACA \\
\hline fimH-R & GTTTTGGCTTTTCGCACAAT \\
\hline \multicolumn{2}{|c|}{ fimS ON/OFF status check } \\
\hline OL4 & CCGTAACGCAGACTCATCCTC \\
\hline OL20 & GAGTTTTAATTTTCATGCTGCTTTCC \\
\hline \multicolumn{2}{|l|}{$\Delta$ fim construction } \\
\hline fimA.ext-5 & CTTGACCTTAATGAAGGTAG \\
\hline fimH.ext-3 & TCCAGCAACTGGTCAGCTGGTT \\
\hline cat.verif-5 & TACGCAAGGCGACAAGGTGC \\
\hline cat.verif-3 & GTACATTGAGCAACTGACTG \\
\hline \multicolumn{2}{|l|}{ PcLfim construction } \\
\hline fimA.ext-5 & CTTGACCTTAATGAAGGTAG \\
\hline fimA.ext-3 & CCGGCAAGTTTCCGCAATAATG \\
\hline spec.verif-5 & CCGATACTCTGAAGCTATGGAACTC \\
\hline spec.verif-3 & ACAAATGCACGGCCAGCAAC \\
\hline \multicolumn{2}{|l|}{ PcLfim verification } \\
\hline fimA.ext-ext-5 & ATGTTTCACTTAGTTTGTTTGCCG \\
\hline fimA-ext-ext-3 & GTTAAAGAGAACCAGGCCTGGG \\
\hline \multicolumn{2}{|c|}{ ecpD/ecp $R$ construction } \\
\hline Ecp-Long_5 & $\begin{array}{l}\text { GATTTTTGGGGCTCTGAGGTGGAATTCTTCCCTCGACCGAA } \\
\text { ATCGCGTAGTTCGTGGCGA }\end{array}$ \\
\hline Ecp-Long_3 & $\begin{array}{l}\text { TGTCCGGCTAAGCCCCCTTCGGCGGACTAAGACTTATCCAG } \\
\text { GTTAGCTCTTTCGCGCCTG }\end{array}$ \\
\hline Ecp_insert_verif_5 & GGTGTGCATATGAGAAAGCCCA \\
\hline Ecp insert_verif 3 & TCGACAAGGTGACAGGAACGG \\
\hline ecpD_mut_verif_5 & ATCTTACAACTTAATCCTGAACGG \\
\hline ecpD_mut_verif_3 & CTATCGATTTTCGCTACAGTGG \\
\hline ecpR_mut_verif_5 & CTTTAATGCCTTCGGCATACTGG \\
\hline ecpR_mut_verif_3 & TATACGCTGGACTGAGTCGTG \\
\hline \multicolumn{2}{|l|}{ RT-PCR } \\
\hline $16 \mathrm{~S} \_\mathrm{F}$ & GTACTTTCAGCGGGGAGG \\
\hline $16 \mathrm{~S} R$ & CAGTTCCCAGGCTGAGC \\
\hline ecpD_F & GCTGGTGGTTACGATATAGCC \\
\hline ecpD_R & CCGGAGTCTGGCTGGTATG \\
\hline
\end{tabular}




\section{SUPPLEMENTARY FIGURES}

$\mathbf{A}$ Populations (biofilm microfermentor assay)

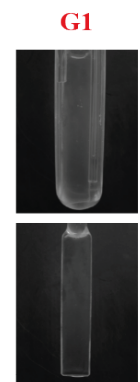
G2

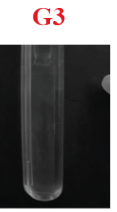

G4 G5
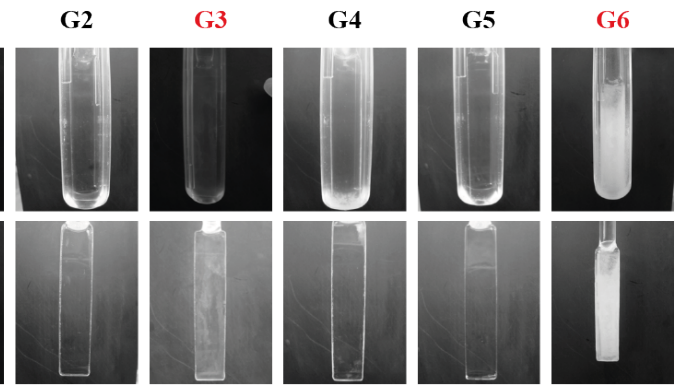

R1

R2

R3

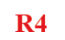

R5

R6
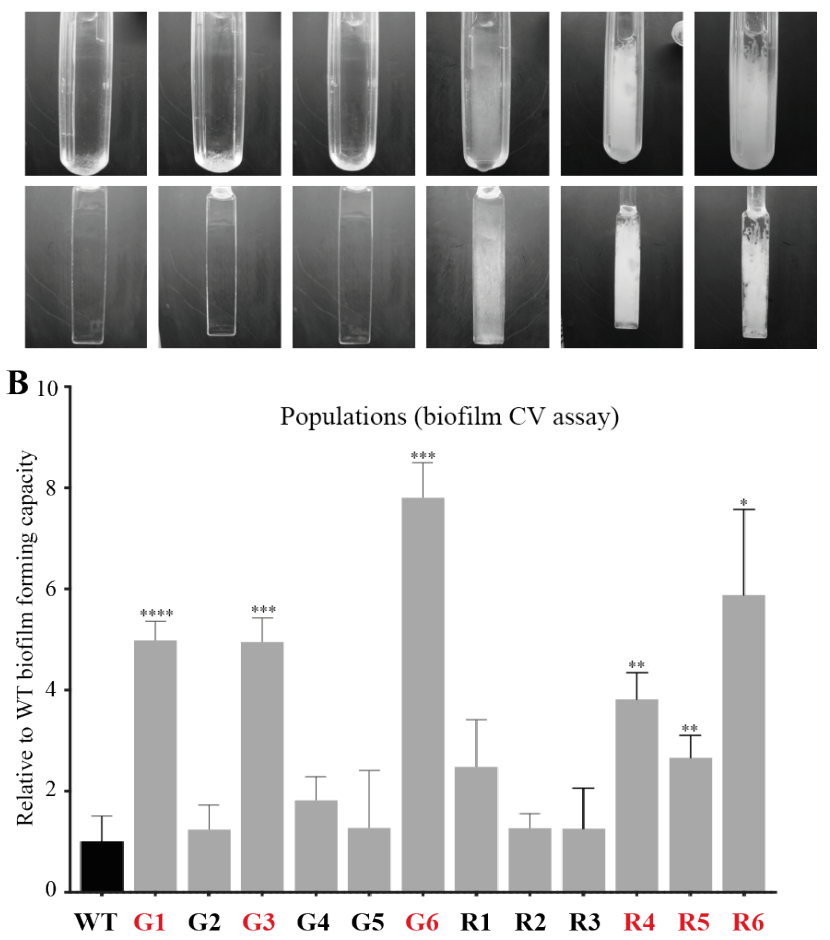

Supplementary Figure S1. Selection of $E$. coli populations with increased biofilm capacity after positive selection. A. Comparison of biofilm formation on microfermenter spatulas of end-point populations after 24 hours. The bottom of the microfermenter (top) and the lower part of the spatula inserted in the microfermenter (bottom) are shown. Selected populations with increased biofilm capacity are indicated in red. B. Comparison of biofilm forming capacities (crystal violet CV biofilm assay) of all populations resulting from positive selection for increased adhesion at cycle 15 . The ancestral wild type is shown in black. The selected populations with increased biofilm capacity are indicated in red. The relative biofilm capacities were calculated using the capacities of the parental wild type trains (set to 1). All CV biofilm assay experiments were conducted with at least 8 replicates. Statistics correspond to unpaired, non-parametric Mann-Whitney test comparing all conditions to WT. *: $\mathrm{p}<0.05 ;{ }^{* *}: \mathrm{p}<0.01 ; * * * \mathrm{p}<0.001 ; * * * *: \mathrm{p}<0.0001$; absence of $*$ : non-significant. 
A

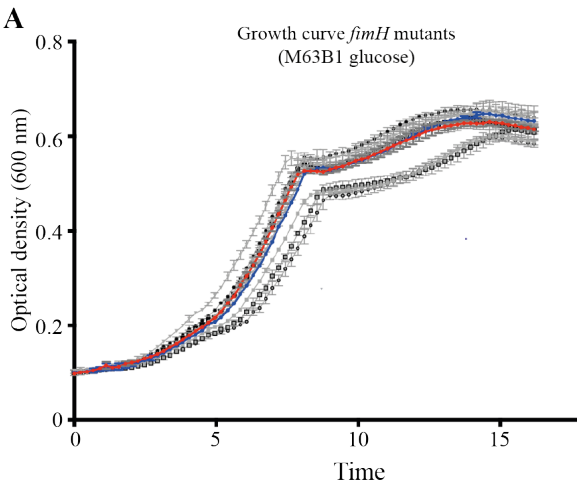

B

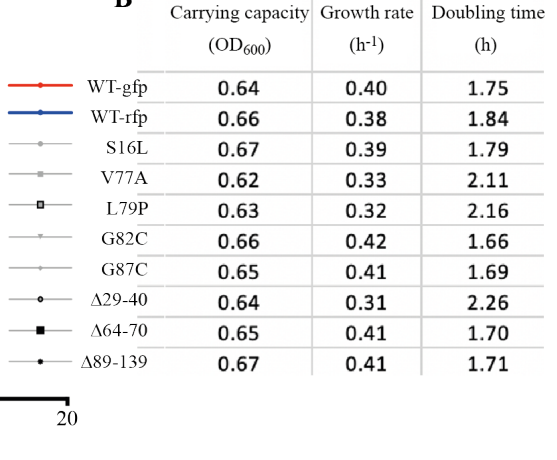

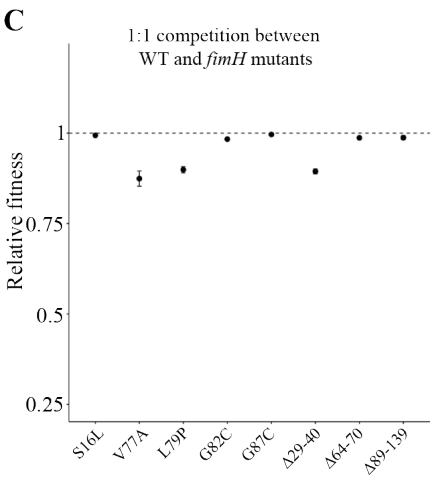

1204

1205 Supplementary Figure S2. Relative growth capacities and fitness of the identified fimH mutants.

1206 A. Growth curves in minimal M63B1 glucose medium of WT ancestors (tagged with GFP or RFP)

1207 together with all identified fim $H$ mutants. B. Carrying capacity, growth rate and doubling time of all

1208 tested strains. The growth was determined by measuring the $\mathrm{OD}_{600}$ using a plate reader. The

1209 experiment was conducted in triplicate. C. Competitions between individual fimH mutants and the WT

1210 ancestor in planktonic culture. The fitness of each mutant is expressed relatively to that of the

1211 ancestor, set to 1 (dashed line).

1212

1213 


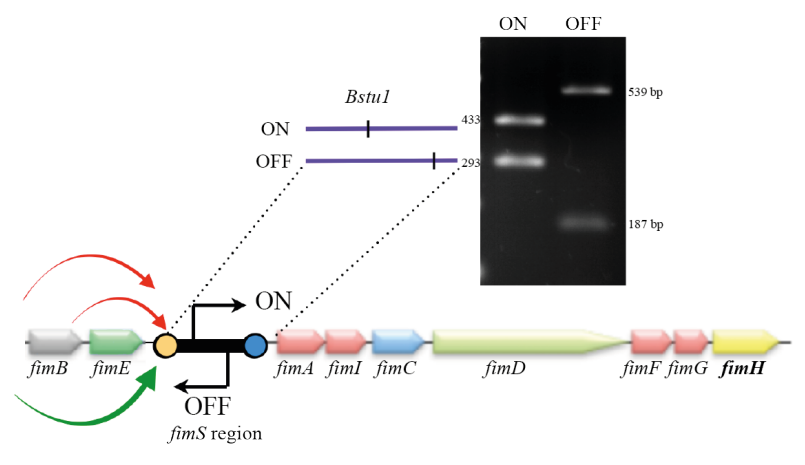

B Biofilm on spatula after $24 \mathrm{H}$ in microfermenter

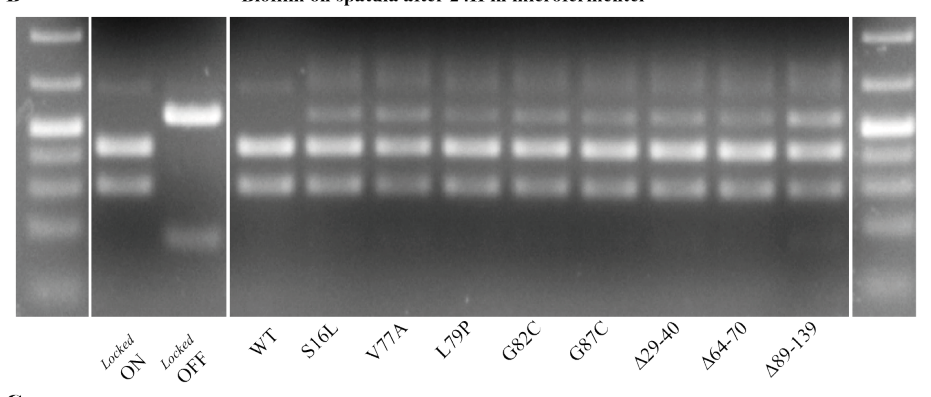

C

$16 \mathrm{H}$ planktonic culture

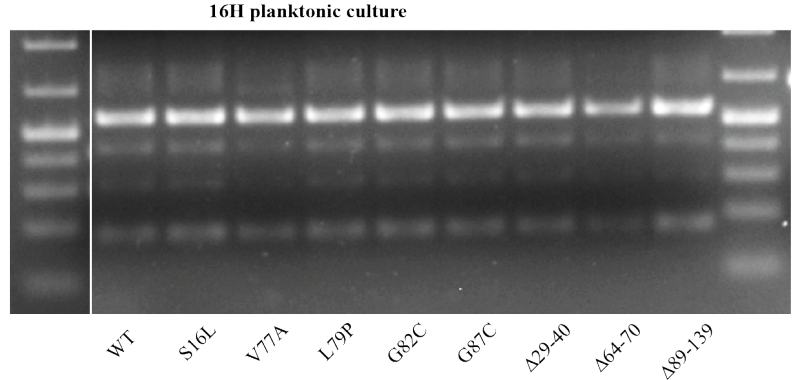

1215 Supplementary Figure S3. Determination of $\mathrm{fim}$ "ON/OFF" status of evolved clones.

A. Genetic organization of the type 1 fimbriae fim operon and representation of the fimS ON-OFF orientation switch controlled by FimB and FimE recombinases. Top: PCR assay followed by BstUI restriction enzyme digestion to distinguish fimS ON and OFF orientation. Phase ON populations of bacteria yielded two DNA fragments 433 and $293 \mathrm{bp}$ in length, whereas phase OFF populations yielded two fragments of 539 and $187 \mathrm{bp}$. B. PCR assay determining the fimS ON and OFF orientation in biofilm population on continuous flow biofilm microfermenter spatula of WT and indicated fim $H$ mutants. C. PCR assay determining the fimA ON and OFF orientation in planktonic population on continuous flow biofilm microfermenter spatula of WT and indicated fim $H$ mutants. Control for OFF state correspond to CJD957= VL386 fimB::Km locked OFF for type 1 fimbriae (1). Control for ON state correspond to CJD808= VL386 fimB::Km locked ON for type 1 fimbriae (2). gene of Escherichia coli is sensitive to changes in DNA supercoiling. Molecular microbiology 14(5):975-988. 
A

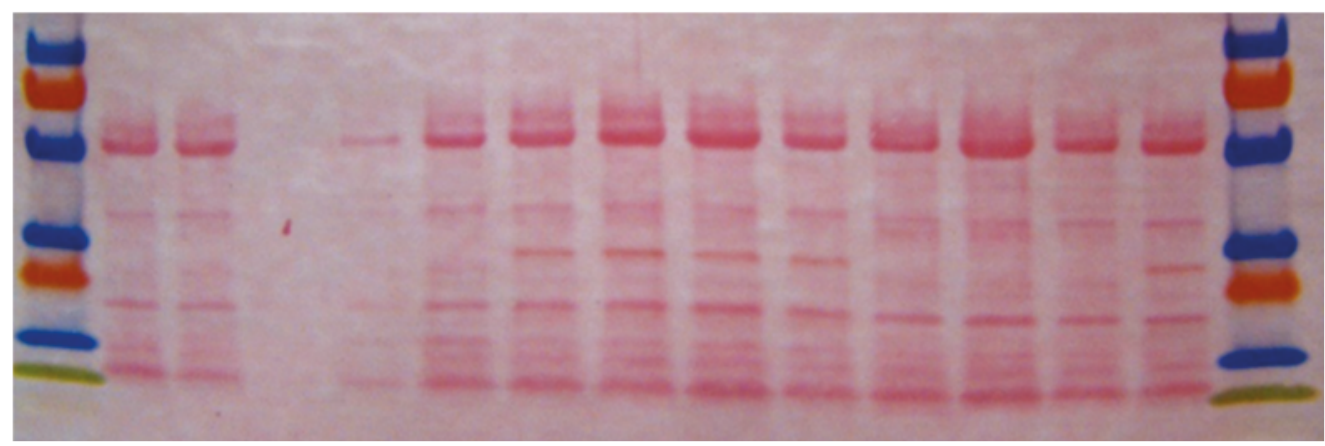

B

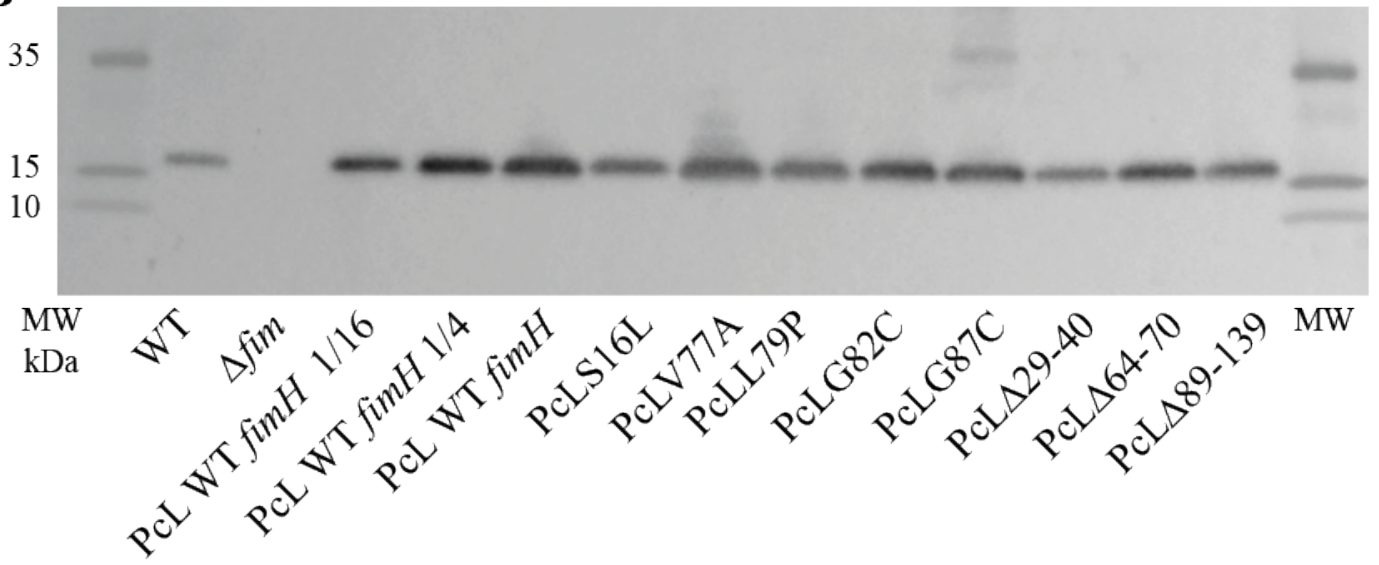

1234 Supplementary Figure S4. Immunodetection of type 1 fimbriae in wild type, $\Delta$ fim, PcLfimH WT,

1235 and PcLfimH mutant strains. A. Ponceau red staining of the nitrocellulose membrane after SDS-

1236 PAGE and transfer showing that $\mathrm{OD}_{600}=2.0$ equivalent of heat extracted proteins were loaded. $\mathbf{B}$.

1237 Immunodetection of type 1 fimbriae (using anti-FimA antibodies) in wild type, $\Delta$ fim, PcLfimH WT,

1238 and PcL fimH mutant strains. 


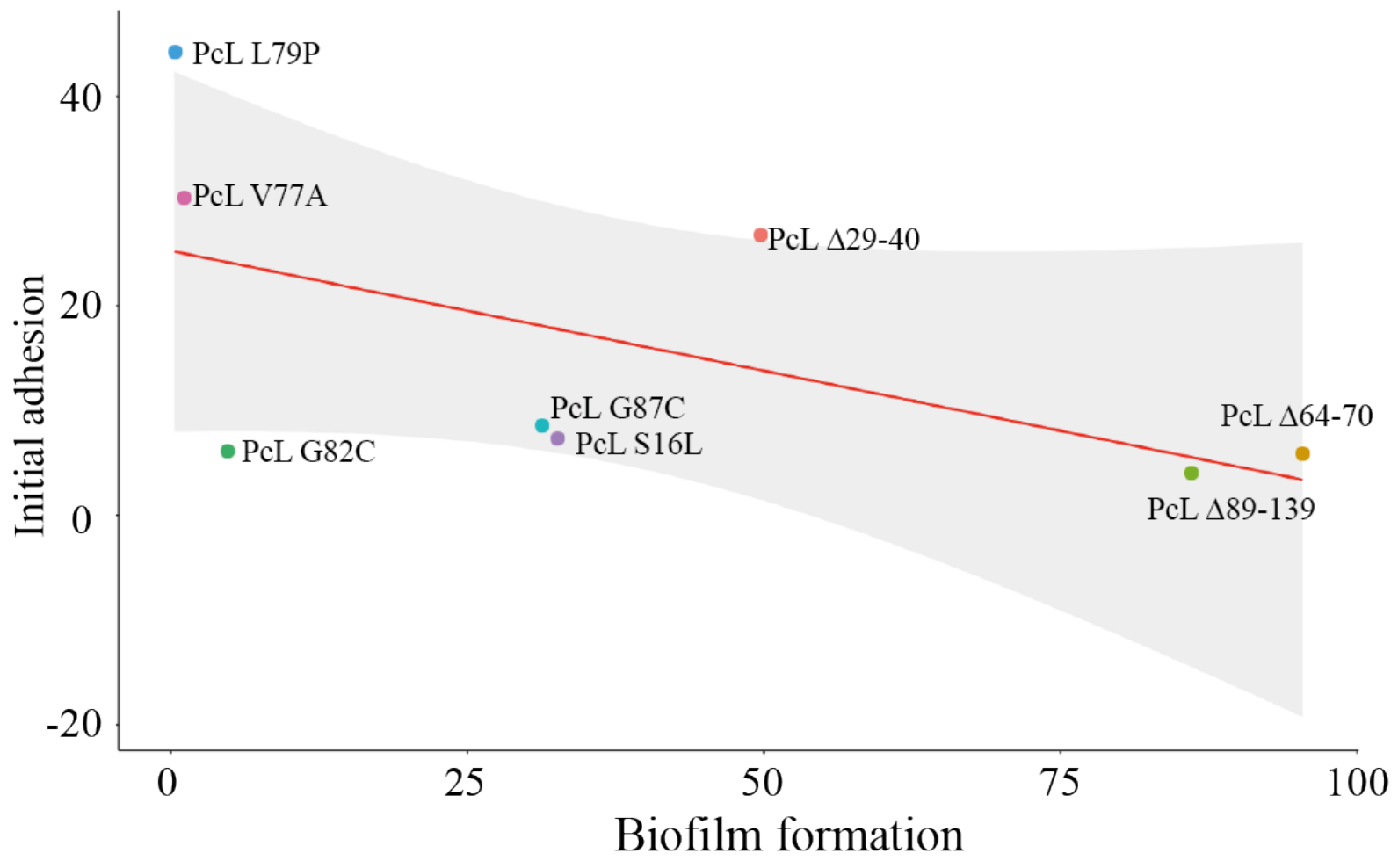

\section{Supplementary Figure S5. Negative trend between initial adhesion and biofilm}

1243 formation in PcLfimH mutants.

1244 Scatter plot showing initial adhesion vs biofilm formation using data from Figure 4. The trend

1245 line is shown in red and the confidence interval in grey. Spearman's rho $=-0.76, p$-value $=$

12460.037.

1247

1248 


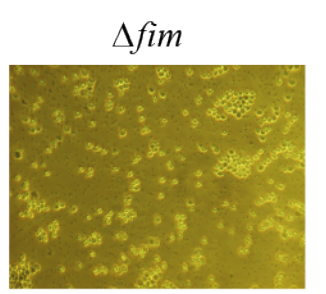

A $\Delta$ fimH
WT

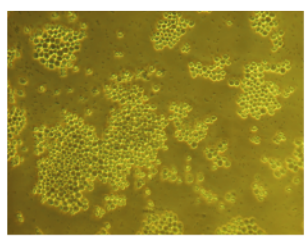

PcLfim

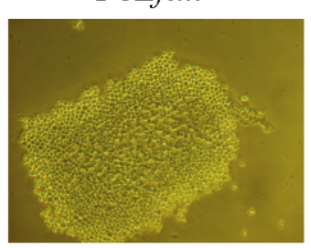

Yeast agglutination in dynamic conditions

\section{fimH WT}

PcL fimH WT

OD600

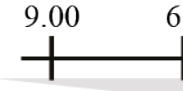

$\Delta 64-70$

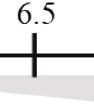

1
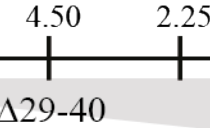

$\Delta 89-139$

$0.56 \quad 0.28$

L79P
0.14

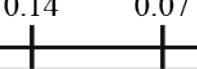

V77A S16L
$0.04 \quad 0.02$

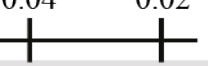

$\mathrm{G} 82 \mathrm{C}$

G87C

B

Yeast agglutination in static conditions

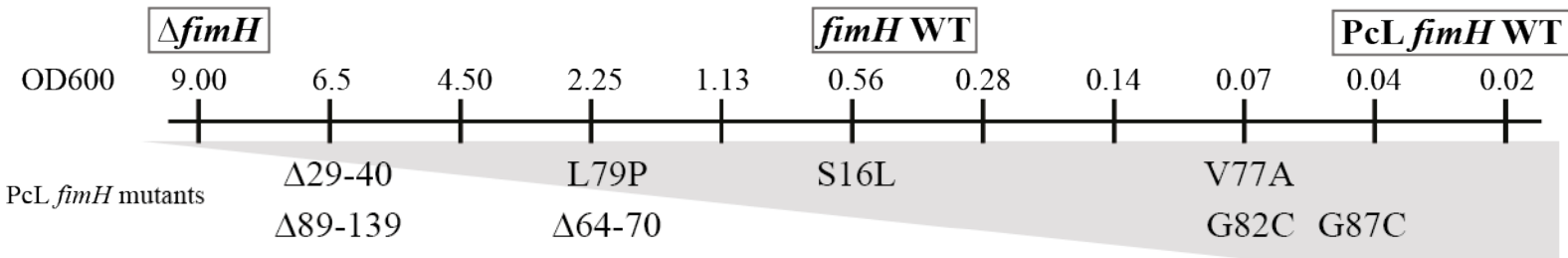

1251 Supplementary Figure S6. Mannose-binding capacity of fim $H$ mutants.

1252 Yeast agglutination of PcL fim $H$ mutants were compared with three control strains $(\Delta$ fim,

1253 wild type WT and PcLfimH WT strains) in dynamic conditions (A) and static conditions (B).

1254 The lowest bacterial concentrations $\left(\mathrm{OD}_{600}\right)$ at which the strains agglutinate with the yeast are

1255 shown: 9.0, 0.56 and 0.04 for $\Delta$ fim, WT and PcLfim $H$ WT strains, respectively. 


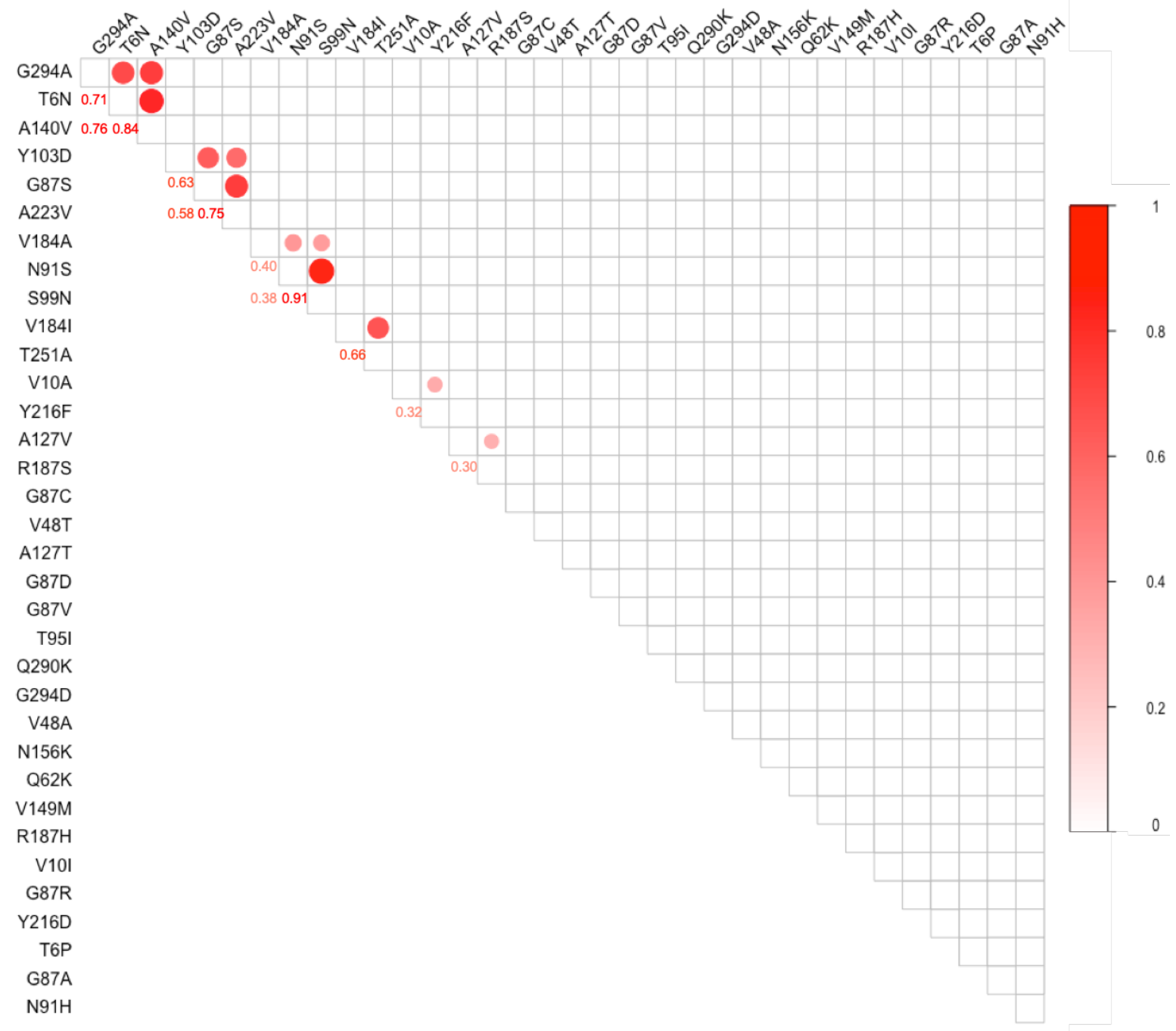

1259 Supplementary Figure S7. Co-occurring mutations at hotspots. All haplotypes are represented on

1260 both axes. On the upper triangle of the matrix, the correlation is represented as a circle if the

1261 correlation is statistically significant ( $\mathrm{p}$-value $<0.05$ ). The color and size of the circle is proportional to the value of the correlation coefficient, which is given on the lower triangle. 


\section{SG4}

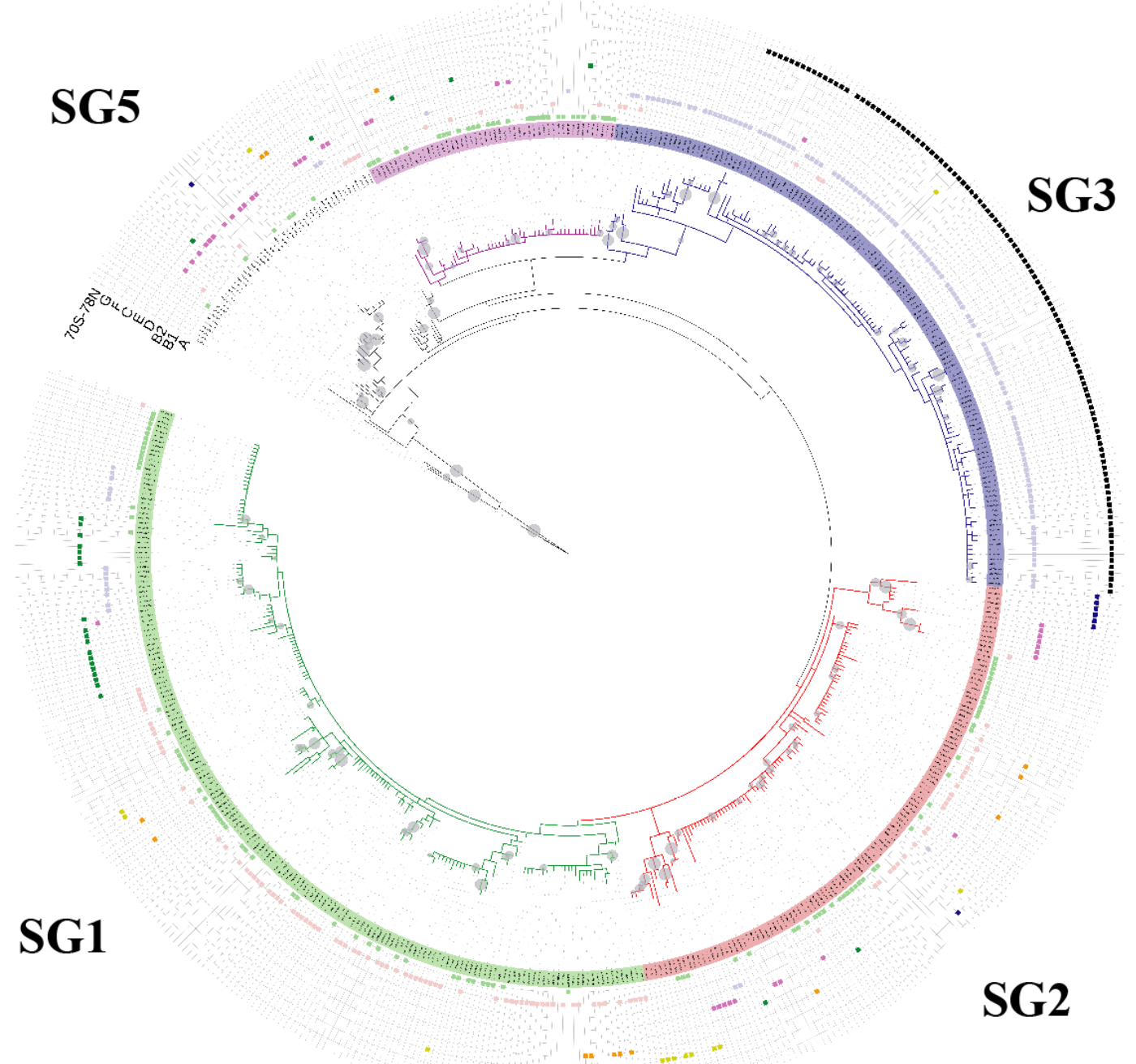

Tree scale: $0.01 \longmapsto$ pressure analysis by PAML. The tree was edited using iTOL v4 ${ }^{74}$. Bootstrap values higher than 0.5 are identified by a grey circle on the corresponding leaves of the tree. The size of the circle is proportional to the bootstrap value. The five sub-groups are represented by different colors ( $\mathrm{Sg} 1 \mathrm{in}$ green, $\mathrm{Sg} 2$ in red, $\mathrm{Sg} 3$ in blue, $\mathrm{Sg} 4$ in purple and $\mathrm{Sg} 5$ in black). Outside the tree, nine additional information are represented. From the inside to the outside: sequences of strains belonging to phylogroup A, B1, B2, D, E, C, F and G are marked with different colored squares. Finally, sequences having $70 \mathrm{~S}$ and $78 \mathrm{~N}$ positions ( $91 \mathrm{~S}$ and $99 \mathrm{~N}$ in this study), which were related to UPEC strains (1), are marked with a black square. addition to mannose binding. Proceedings of the National Academy of Sciences of the United States of America 106(52):22439-22444. 


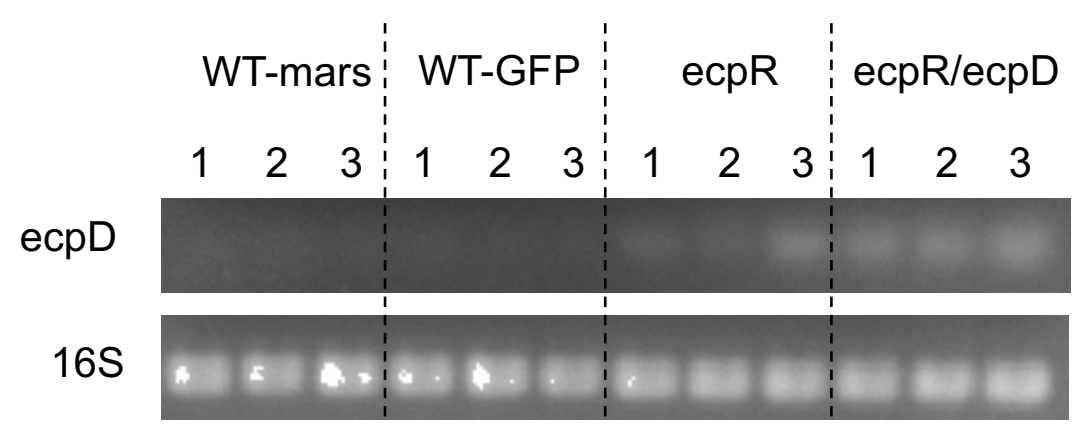

\section{Supplementary Figure S9. Impact of IS2 insertion on ecp operon expression.}

1290 RT-PCR targeting ecpD in WT strain with either mars or GFP marker as well as in strains

1291 selected in the $\Delta$ fim evolution and having the IS2 insertion after ecpR. 16S rRNA is used as a

1292 reference. For each strain, the three biological replicates are marked with numbers.

1293

1294

1295

1296

1297

1298

1299

1300

1301

1302

1303 

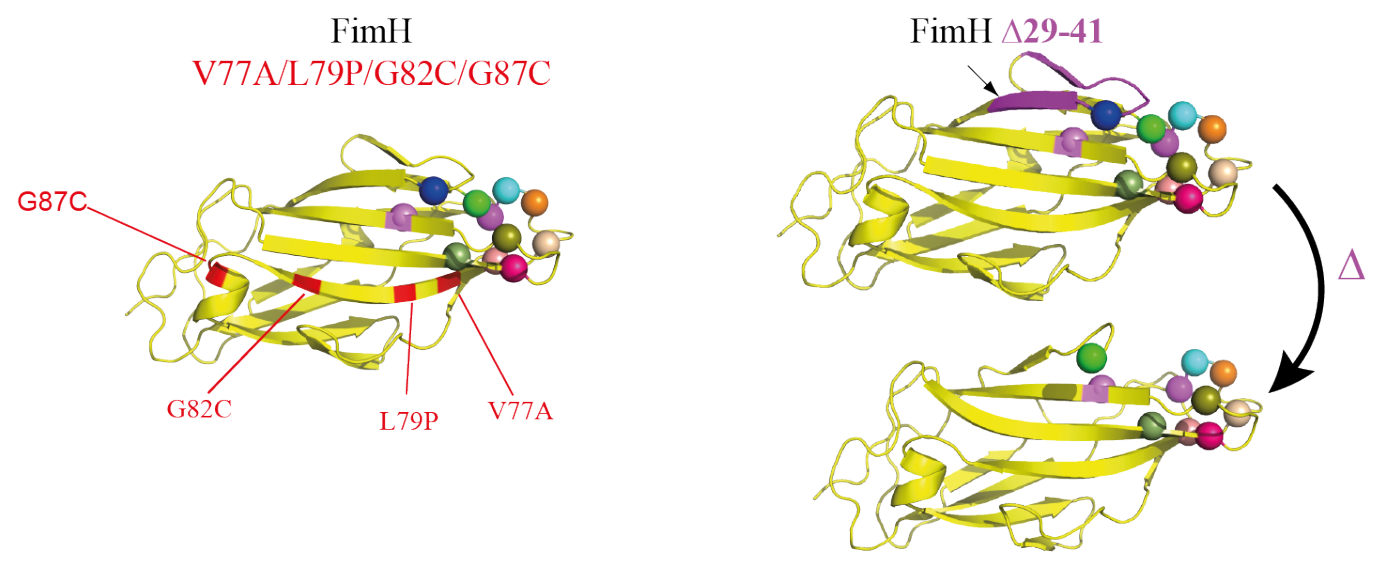

FimH $\Delta 64-70$

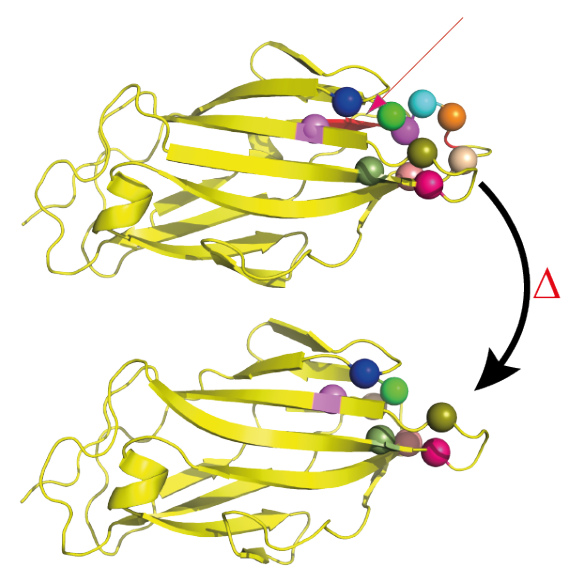

FimH $\triangle 89-139$

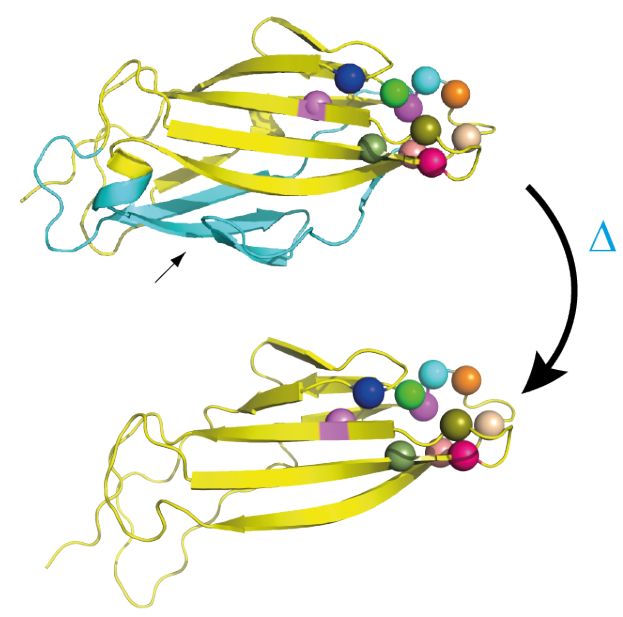

Supplementary Figure S10. Structural changes in the FimH lectin domain with selected

mutations or deletions. These positions are compared in relation to the amino-acids residues forming the mannose binding pocket and the surrounding hydrophobic ridge (represented by spheres). For the three deletions isolated in our study, the potential structural modification of the deletion have been modelled using Phyre2 (1). The modification of the presence or the organisation of these important residues is shown upon the three deletions that affected the most strongly FimH mannose-binding capacity. protein modeling, prediction and analysis. Nature Protocols 10:845.

1316 